\title{
Mapping of Extrinsic Innervation of the Gastrointestinal Tract in the Mouse Embryo
}

\author{
Xueyuan Niu, ${ }^{\star}$ Li Liu, ${ }^{\star}$ Tao Wang, Xin Chuan, Qi Yu, Mengjie Du, Yan Gu, and ${ }^{\circledR}$ Liang Wang \\ Institute of Neuroscience and Department of Neurology of the Second Affiliated Hospital, Mental Health Center, NHC and CAMS Key Laboratory of \\ Medical Neurobiology, Zhejiang University School of Medicine, Hangzhou 310058, China
}

Precise extrinsic afferent (visceral sensory) and efferent (sympathetic and parasympathetic) innervation of the gut is fundamental for gut-brain cross talk. Owing to the limitation of intrinsic markers to distinctively visualize the three classes of extrinsic axons, which intimately associate within the gut mesentery, detailed information on the development of extrinsic gut-innervating axons remains relatively sparse. Here, we mapped extrinsic innervation of the gut and explored the relationships among various types of extrinsic axons during embryonic development in mice. Visualization with characterized intrinsic markers revealed that visceral sensory, sympathetic, and parasympathetic axons arise from different anatomic locations, project in close association via the gut mesentery, and form distinctive innervation patterns within the gut from embryonic day (E)10.5 to E16.5. Genetic ablation of visceral sensory trajectories results in the erratic extension of both sympathetic and parasympathetic axons, implicating that afferent axons provide an axonal scaffold to route efferent axons. Coculture assay further confirmed the attractive effect of sensory axons on sympathetic axons. Taken together, our study provides key information regarding the development of extrinsic gut-innervating axons occurring through heterotypic axonal interactions and provides an anatomic basis to uncover neural circuit assembly in the gut-brain axis (GBA).

Key words: axon-axon interaction; extrinsic innervation; spatiotemporal development; the gastrointestinal tract; visceral motor efferent; visceral sensory afferent

Significance Statement

Understanding the development of extrinsic innervation of the gut is essential to unravel the bidirectional neural communication between the brain and the gut. Here, with characterized intrinsic markers targeting vagal sensory, spinal sensory, sympathetic, and parasympathetic axons, respectively, we comprehensively traced the spatiotemporal development of extrinsic axons to the gut during embryonic development in mice. Moreover, in line with the somatic nervous system, pretarget sorting via heterotypic axonal interactions is revealed to play critical roles in patterning extrinsic efferent trajectories to the gut. These findings provide basic anatomic information to explore the mechanisms underlying the process of assembling neural circuitry in the gut-brain axis (GBA).

\section{Introduction}

The gastrointestinal (GI) tract is innervated both intrinsically by the enteric nervous system (ENS), and extrinsically by visceral

\footnotetext{
Received Feb. 2, 2020; revised June 5, 2020; accepted July 12, 2020.

Author contributions: L.W. and X.N. designed research; X.N., L.L., T.W., X.C., Q.Y., M.D., and Y.G. performed research; Y.G. and L.W. analyzed data; L.W. wrote the paper.

${ }^{*}$ X.N. and L.L. contributed equally to this work.

This work was supported by the National Key R\&D Program of China Grant 2017YFA0104200, the Zhejiang Provincial Natural Science Foundation of China Grant LY19C090005, the Fundamental Research Funds for the Central Universities Grant 2019FZA7009, and the Chinese Ministry of Education Project 111 Program Grant B13026. We thank members of the core facilities of the Institute of Neuroscience, Zhejiang University, and members of the core facilities of Zhejiang University School of Medicine for their outstanding technical support. We appreciate critical comments on our manuscript from W. Y. Chan. We further thank G. Schütz, F. Wang, and S. L. Pfaff for providing $D b h^{\text {(re }}$ mice, Advillin ${ }^{C r e}$ mice, and $H b 9^{G F P}$ mice, respectively.

The authors declare no competing financial interests.

Correspondence should be addressed to Liang Wang at Iwang1@zju.edu.cn.

https://doi.org/10.1523/JNEUROSCI.0309-20.2020

Copyright $\odot 2020$ the authors
}

sensory afferent and visceral motor efferent (sympathetic and parasympathetic) fibers. The ENS is composed of millions of neurons and glia within the gut to control GI functions including gut motility and digestive secretion (Obermayr et al., 2013a; Rao and Gershon, 2018). Precise extrinsic innervation of the GI tract is critical for both gut homeostasis and brain emotional and cognitive functions through bidirectional communication between the gut and the brain (Dinan and Cryan, 2017). In contrast to considerable advances in mapping neural circuitry along the gut-brain axis (GBA) within adult animals (Han et al., 2018; Kaelberer et al., 2018), relatively little is known about the mechanisms underlying visceral nerve assembly to integrate visceral sensory afferent and motor efferent axons into common pathways during embryonic development.

The GI tract receives dual sensory afferent innervation, with cell bodies located in the nodose ganglia (NGs), the inferior ganglion of the vagus nerve, and dorsal root ganglia (DRGs), to 
convey mechanical and chemical information of the gut to the brain (Brookes et al., 2013). Sympathetic and parasympathetic nerves are integrated with visceral sensory pathways to transmit efferent signals from the brain to the gut. Sympathetic preganglionic neurons, residing within the thoracolumbar spinal cord, make synaptic connections with postganglionic neurons in the prevertebral ganglion (PVG) and pelvic ganglion (PG), which in turn innervate the GI tract. By contrast, parasympathetic preganglionic neurons, originating from the dorsal motor nucleus of the vagus nerve (DMN) in the hindbrain and the intermediolateral column of the sacral spinal cord, extend projections to form synapses with postganglionic neurons within the bowel and the PG, respectively (Browning and Travagli, 2014). Previous studies with various neural tracers (Rinaman and Levitt, 1993; Ratcliffe et al., 2006; Murphy and Fox, 2007; Wang and Powley, 2007; Powley et al., 2011), together with immunostaining (Tassicker et al., 1999; Tan et al., 2010; Hatch and Mukouyama, 2015), have preliminarily mapped the timelines of extrinsic axons to the gut. However, the inherent limitations of neural tracers, including slow diffusing rate, short tracing distance, unstable labeling efficiency, and tracer leakage, potentially lead to incomplete and/or false-positive interpretations. Moreover, the lack of intrinsic markers impedes our ability to distinguish or manipulate distinctive extrinsic axons for further mechanistic investigations. In spite that pretarget sorting via axon-axon interaction has been implicated in the topographic mapping of various types of axons (Imai et al., 2009; Wang et al., 2011; Wang and Marquardt, 2013; Cioni et al., 2018), insights into the roles of pretarget sorting of extrinsic gut-innervating axons are sparse. A previous study has shown that visceral afferents of the head provide a scaffold for parasympathetic efferent axons (Coppola et al., 2010), whether this heterotypic axonal interaction is conserved within the vagus nerves remains to be addressed. Therefore, the present study was designed to map extrinsic innervation of the GI tract on an organ-wide scale with the following aims: (1) identify specific intrinsic markers to simultaneously visualize sensory afferent, sympathetic efferent, and parasympathetic efferent axons; (2) spatiotemporally map the timeline of extrinsic innervation of the gut with newly identified intrinsic markers; and (3) determine the relationship among various types of gut-innervating extrinsic axons.

\section{Materials and Methods}

Mice

The procedures for all mouse experiments conformed to the regulations set by the Animal Care and Use Committee of Zhejiang University. Advillin $^{\text {Cre }}$ (Hasegawa et al., 2007), Hb9 ${ }^{G F P}$ (Lee et al., 2004), Dbh Cre (Parlato et al., 2007), v Glut2 $^{\text {Cre }}$ (JAX 016963), Chat ${ }^{\text {Cre }}$ (JAX 006410), Chat $^{\text {GFP }}$ (JAX 007902), Npy ${ }^{\text {hrGFP }}$ (JAX 006417), Vip ${ }^{\text {Cre }}$ (JAX 010908), R26 $6^{\text {lsl-tdTomato }}$ (JAX 007909), Phox $2 b^{F L P}$ (JAX 022407), R26 $6^{\text {FLTG }}$ (JAX 026932), and R26 $6^{\text {lsl-DTA }}$ (JAX 006331) mouse lines have been described previously. Mice were either maintained independently or interbred to label or ablate a defined population of neurons. The morning a vaginal plug was found in mice was designated as embryonic day (E)0.5.

\section{Immunohistochemistry}

Immunofluorescence staining was performed as described previously (Wang et al., 2011). The following primary antibodies were used in this study: rabbit anti-GFP (1:3000, Invitrogen, A11122); chicken anti-GFP (1:3000, Abcam, ab13970); rabbit anti-RFP (1:2000, Abcam, ab62341); rat anti-RFP (1:1000, Chromotek, 5F8); goat anti-mCherry (1:1000, SICGEN, AB0040-200); chicken anti-dsRed (1:2000, EnCor Biotechnology Inc, CPCA-mcherry); mouse anti-Tuj1 (1:3000, Abcam, ab78078); rabbit anti-TH (1:1500, Millipore, 657012); goat anti-TH
(1:1000, Millipore, AB1542); rabbit anti-VAChT (1:2000, SYSY, 139103); guinea pig anti-VAChT (1:1000, SYSY, 139105); rat anti-PECAM (1:4000, BD PharMingen, 553371); rabbit anti-TrkA (1:500, Millipore, 06-574); goat anti-Phox2b (1:500, R\&D Systems, AF4940); rabbit antiBrn3a (1:1000, SYSY, 41103). Secondary antibodies used were Alexa Fluor 405, Alexa Fluor 488, Alexa Fluor 555, and Alexa Fluor 637 (Life Technologies, all at 1:1000). For section immunofluorescence staining, sections were incubated in primary antibodies for $20 \mathrm{~h}$ at room temperature (RT), then secondary antibodies for $1 \mathrm{~h}$ at RT. For whole-mount immunofluorescence staining, tissues were flat-mounted in silicone dissecting dishes (prepared with Sylgard kit; WPI) with Minutien Pins (F.S. T.), and then fixed in 4\% paraformaldehyde (PFA)/PBS for 6-24 h according to tissue sizes at RT. The tissues were further fixed with Dent's solution (Methanol:DMSO 4:1) for 3-24 h. Primary antibodies and secondary antibodies were incubated for 36-48 and 12-24 h, respectively. After washing, the samples were cleared with BABB solution (benzyl alcohol:benzyl benzoate 1:2; Sigma) for imaging.

\section{In vivo DiI tracing}

DiI (Sigma) dissolved in methanol was injected into different regions of the GI tract. Embryos were then incubated in $4 \%$ PFA/PBS at $37^{\circ} \mathrm{C}$ for one to two weeks to allow sufficient diffusion of DiI.

\section{Sympathetic-sensory coculture assay}

Fluorescence-guided microdissection and coculture assay were performed as described previously (Wang and Marquardt, 2012). Briefly, PVGs from E14.5 Npy ${ }^{h r G F P}$ embryos and NGs or DRGs from E14.5 $v$ Glut $2^{\text {Cre }} ; R 26^{\text {tdTom }}$ were placed at 200 - to $500-\mu \mathrm{m}$ distance on poly-D-lysine/laminin double-coated glass coverslips, followed by cultured in neurobasal medium containing various growth factors. NGF (R\&D Systems, 256-GF) was applied with the concentration of $100 \mathrm{ng} \mathrm{ml}^{-1}$ for PVG and DRG culture, and BDNF (R\&D Systems, 248-BD) was applied with the concentration of $50 \mathrm{ng} \mathrm{ml}^{-1}$ for NG culture.

\section{Microscopy and image analysis}

Images were collected on an Olympus Fluoview FV3000 confocal microscope (Olympus). Whole embryo and gut images were obtained by tile scanning with a $10 \times$ objective and automatic stitching. Three-dimensional reconstructions were obtained with IMARIS imaging software (Bitplane). Images were processed and analyzed with Adobe Photoshop (Adobe) and Image (National Institutes of Health).

\section{Experimental design and statistical analyses}

Statistical analysis was performed using GraphPad Prism v7.0 (GraphPad Software). The choice of specific statistical test was based on the distribution of each group. If normality assumptions were met, $t$ test was used. Otherwise, Kruskal-Wallis test was used. ImageJ software was used for the quantification of fluorescence intensity and neuron numbers. Consecutive $30-\mu \mathrm{m}$-thick sections of embryos from various developmental stages were collected to score the number of immunodetected neurons in the peripheral ganglia. For each experimental group, three or four embryonic samples from at least two pregnant mice were analyzed. Data were presented as means \pm SEM, and $p<0.05$ was considered significant. Detailed statistical information for $\mathrm{n}$ and $\mathrm{p}$ value for each experiment was written in the corresponding figure legends.

\section{Results}

Tracing extrinsic innervation of the GI tract by three types of axons

To determine the time point when extrinsic axons entered the gut, the entire GI tract was stained with pan-neuronal marker $\beta$ III-tubulin (Tuj1) and ENS marker Phox2b. At E14.5 after the gut had been fully colonized by enteric neural crest cells (NCCs), the stomach, foregut, and proximal part of the midgut were innervated by extrinsic fibers, whereas most extrinsic axons were still under their projection pathways through the gut mesentery (Fig. 1A). By E16.5, extrinsic axons were found along the whole gut and mixed with intrinsic enteric axons (Fig. 1B). To identify 


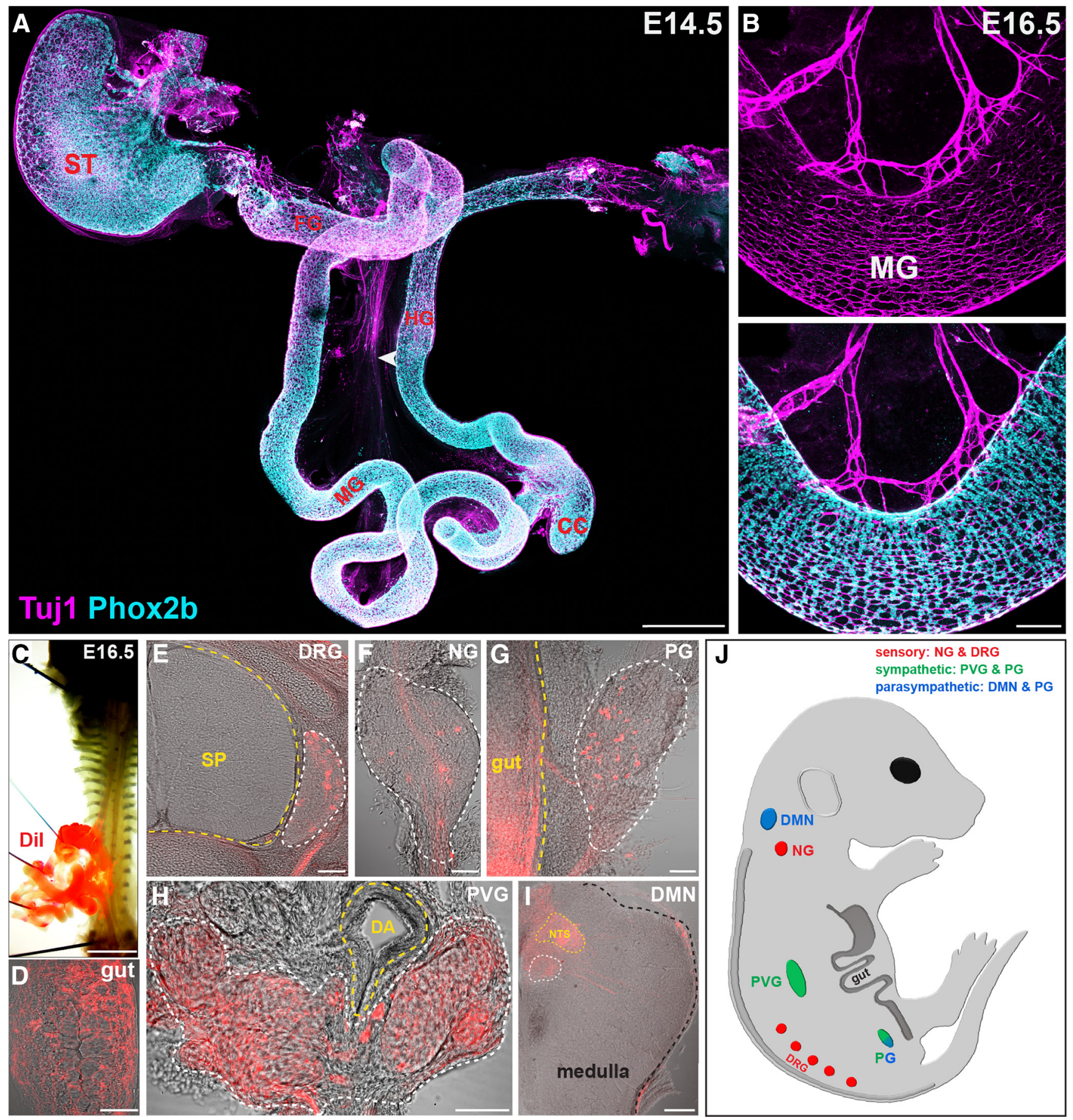

Figure 1. Three sources of extrinsic gut-innervating axons. $A$, Representative whole-mount view of the gut from E14.5 embryos $(n=3)$ with Tuj1 and Phox2b staining. Arrowhead indicates pioneer axons within the gut mesentery. $\boldsymbol{B}$, Representative midgut segment of E16.5 embryos $(n=3)$. C, Representative sample of Dil-labeled E16.5 guts $(n=3)$. D, Image of Dil-labeled midgut segment from E16.5 embryos $(n=3)$. $\boldsymbol{E}-\boldsymbol{I}$, Transversal sections from the sample shown in $\boldsymbol{C}$ demonstrating the presence of Dil ${ }^{+}$cells in the DRG, NG, PG, PVG, and DMN, respectively. J, Schematic representation of the three sources of extrinsic innervation of the gut. Scale bars: $400 \mu \mathrm{m}(\boldsymbol{A}), 100 \mu \mathrm{m}(\boldsymbol{B}, \boldsymbol{D}, \boldsymbol{E}-\boldsymbol{H}), 2 \mathrm{~mm}(\boldsymbol{C}), 200 \mu \mathrm{m}(\boldsymbol{I})$. ST, stomach; FG, foregut; MG, midgut; CC, caecum; HG, hindgut; DRG, dorsal root ganglion; NG, nodose ganglion; DMN, dorsal motor nucleus of the vagus nerve; PVG, prevertebral ganglion; PG, pelvic ganglion; SP, spinal cord; DA, dorsal aorta; NTS, nucleus of the solitary tract.

the locations of extrinsic neurons, DiI retrograde tracing was performed for the entire E16.5 gut, followed by histologic analyses (Fig. 1C,D). Three types of DiI-marked neurons were observed in the following locations: (1) sensory neurons in the NG and the DRG; (2) sympathetic neurons in the PVG and the PG; and (3) parasympathetic neurons in the DMN and the PG (Fig. 1E-I). Taken together, these results confirm previous findings that the gut is innervated by sensory afferent, sympathetic efferent, and parasympathetic efferent axons (Brookes et al., 2013; Browning and Travagli, 2014), and these axons extend into the gut by E16.5 (Fig. 1J).

Visualizing and tracing visceral sensory axons to the GI tract The presence of three types of extrinsic axons prompted us to distinguish them with distinctive intrinsic markers for further stereotypic analyses. Reliable markers must satisfy two basic 
criteria: high labeling specificity and efficiency to ensure all targeting neurons to be specifically and completely labeled. To this end, we first assessed transgenic markers in two general sensory neuron reporter lines, i.e., $v$ Glut $2^{\text {Cre }} ; R 26^{\text {tdTom }}$ and Advillin ${ }^{\text {Cre }} ;$ R26 $^{\text {tdTom }}$ (Hasegawa et al., 2007; Chang et al., 2015). In contrast to the observations made in Advillin ${ }^{\text {Cre }} ; R 26^{\text {tdTom }}$ embryos, in which only a subset of sensory neurons was labeled, tdTom fluorescence was exclusively present in the majority of sensory neurons in the NGs and DRGs, but not in sympathetic and parasympathetic neurons, of $v$ Glut $2^{\mathrm{Cre}} ; R_{2} 6^{\text {tdTom }}$ embryos (Fig. $2 A-F$ ), indicating the vGlut ${ }^{C r e} ; R 26^{\text {tdTom }}$ transgene was expressed more specifically and abundantly in early sensory neurons. Thus, the $v$ Glut $2^{\text {Cre }} ; R 26^{\text {tdTom }}$ line was used to trace gutinnervating visceral sensory axons.

Previous tracing studies have shown that visceral sensory neurons convey gut information to the brain through three pathways: vagal (the vagus nerves), splanchnic spinal (the mesenteric nerves and thoracolumbar splanchnic nerves), and sacral spinal (the pelvic and pudendal nerves) afferents with their cell bodies located within the NG, thoracolumbar DRGs, and lumbosacral DRGs, respectively (Brookes et al., 2016). We found that NG sensory neurons in $v$ Glut $2^{\text {Cre }} ; R 26^{\text {tdTom }}$ embryos projected their axons along the esophagus at E10.5 (Fig. 2G, $G$ '), and entered the stomach by E11.5 (Fig. $2 H$ ). One day later at E12.5, vagal afferent fibers had split into two trajectories: gastric branch to the stomach and celiac branch to the intestine via the gut mesentery. The first wave of celiac projections was found to just reach the ventral part of the PVG, where a few spinal sensory axons were also located (Fig. 2I). Meanwhile, sacral spinal sensory axons from the lumbosacral DRGs projected through the PG and extended into the distal hindgut (Fig. 2I'). Thoracolumbar DRG-arising sensory axons first extended ventrally to the paravertebral sympathetic chains, then descended or ascended along the chains, and finally left the sympathetic chains to project to the gut through the PVG (Fig. 2J). The majority of the gut was innervated in a rostral-to-caudal order with the rostral part being first innervated at E14.5, whereas sacral spinal axons extended proximally along the hindgut toward the caecum (Fig. 2J'). At E16.5, while a few scattered $v$ Glut $2^{\text {tdTom }+}$ neurons occurred within the gut, their processes were restricted within the gut wall, therefore making it possible for us to trace extrinsic sensory axons into the gut. Sensory axons projected into the whole gut from the mesenteric side by E16.5 (Fig. 2K).

\section{Distinguishing vagal and spinal sensory trajectories to the GI tract}

We next sought to distinguish vagal sensory and spinal sensory afferent axons to simultaneously map their projection patterns. Different from NCC-derived DRG sensory neurons, NG sensory neurons are derived from epibranchial placodes and could be distinguished from DRG sensory neurons by Phox2b expression (D'Autreaux et al., 2011). Based on this, we generated Phox $2 b^{F L P}$; $v$ Glut $2^{\text {Cre }} ; R 26^{\text {FLTG }}$ transgenic mice, in which Phox $2 b^{+} / v G l u t 2^{+}$ NG sensory neurons were labeled with GFP through FLP-FRT and Cre-loxp double recombination systems (Fig. 3A; Hirsch et al., 2013; Plummer et al., 2015). Consistent with the patterns of endogenous Phox $2 \mathrm{~b}$ and vGlut 2 expression, GFP was exclusively expressed in NG sensory neurons, without any expression in DRG sensory neurons. Meanwhile, sympathetic and parasympathetic neurons were genetically labeled with tdTom after FLPFRT single recombination (Fig. $3 B, C$ ). Previous studies have shown that NG sensory neurons are not responsive to NGF (Lindsay and Rohrer, 1985), suggesting that NGF receptor TrkA might not be expressed in NG sensory neurons. In line with this speculation, we found that TrkA was exclusively expressed in DRG sensory neurons, but not in NG sensory, sympathetic, or parasympathetic neurons during early developmental stage (Fig. $3 D-F)$. Therefore, we performed TrkA staining on the gut from E14.5 Phox $2 b^{F L P} ; v G l u t 2^{C r e} ; R 26^{F L T G}$ embryos to simultaneously trace vagal $\left(\mathrm{GFP}^{+}\right)$and spinal $\left(\mathrm{TrkA}^{+}\right)$sensory axons. Vagal sensory axons descended along the esophagus to innervate the foregut, midgut, caecum, and rostral hindgut (Fig. 3G). Most $\mathrm{TrkA}^{+}$splanchnic spinal axons projected ventrally through the PVG and bifurcated into gastric and celiac trajectories (Fig. 3G, $\left.G^{\prime}\right)$. We noticed that some $\operatorname{TrkA}^{+}$splanchnic spinal axons crossed the PVG caudally to join sacral spinal axons. At the sacral level, sacral and splanchnic spinal axons extended through the PG to innervate the hindgut (Fig. $3 G$ ).

Collectively, our findings demonstrate that vagal, splanchnic spinal, and sacral spinal sensory axons, arising from the NG, thoracolumbar DRGs, and lumbosacral DRGs, respectively, project along defined pathways to innervate the GI tract from E10.5 to E16.5 (Fig. 3H).

\section{Visualizing and tracing sympathetic projection to the GI tract}

Postganglionic sympathetic neurons within the PVG received synaptic input from preganglionic motor neurons within the spinal cord and provided sympathetic innervation of the gut (Fig. $\left.4 A, A^{\prime}\right)$. Given that the primary neurotransmitter of postganglionic sympathetic neurons is norepinephrine, we first characterized the labeling specificity of $D b h^{C r e} ; R 26^{t d T o m}$ line for sympathetic neurons. Unexpectedly, a subset of NG sensory neurons also expressed strong tdTom during early developmental stages (Fig. $4 B-D$ ), thus excluding the possibility to use $D b h^{C r e}$; $R 26^{t d T o m}$ line to distinguish sympathetic and vagal sensory axons. We next examined two other transgenic mouse lines for marking sympathetic neurons: $N p y^{h r G F P}$ and Vip ${ }^{C r e} ; R 26^{\text {tdTom }}$ (Duong et al., 2002; van den Pol et al., 2009) and found that only subsets of sympathetic neurons were genetically labeled in E14.5 Npy ${ }^{h r G F P}$ and Vip ${ }^{C r e} ; R 26^{\text {tdTom }}$ embryos (Fig. $4 E, F$ ). Moreover, subpopulations of enteric neurons in $N p y^{\text {hrGFP }}$ and Vip Cre $; 226^{\text {tdTom }}$ embryos were also genetically labeled (data not shown), making it impossible to separate sympathetic axons from enteric neurons. Therefore, we employed the classic sympathetic neuron marker $\mathrm{TH}$ antibody to trace sympathetic axons.

At E10.5 when sympathetic chains were being formed, scattered NCC-derived $\mathrm{TH}^{+}$neurons were detected along the esophagus and proximal GI tract (Fig. 4G, $G^{\prime}$ ). PVG sympathetic neurons initiated their axons at E12.5 after the sympathetic chains were fully developed. While $\mathrm{TH}^{+}$neurons were present within the PG, no detectable axons were found within the hindgut at E12.5 (Fig. 4H, $H^{\prime}$ ). By E14.5, PVG sympathetic axons split into gastric and celiac trajectories, and PG-arising sympathetic axons projected proximally along the hindgut toward the caecum (Fig. 4I, $I^{\prime}$ ). At this stage, enteric $\mathrm{TH}^{+}$neurons were almost undetectable along the gut, an observation consistent with previous reports that enteric $\mathrm{TH}^{+}$neurons were present transiently (Young et al., 2002). By E16.5, sympathetic axons extended into the whole length of the gut and mixed with enteric neurons (Fig. 4J).

In summary, sympathetic neurons located in the PVG and PG initiate their axons toward the gut at E12.5, and sympathetic axons are found throughout the whole gut by E16.5. The GI tract is mainly innervated by PVG-arising sympathetic axons, with the hindgut being innervated by both PVG and PG-arising sympathetic axons (Fig. $4 K$ ). 

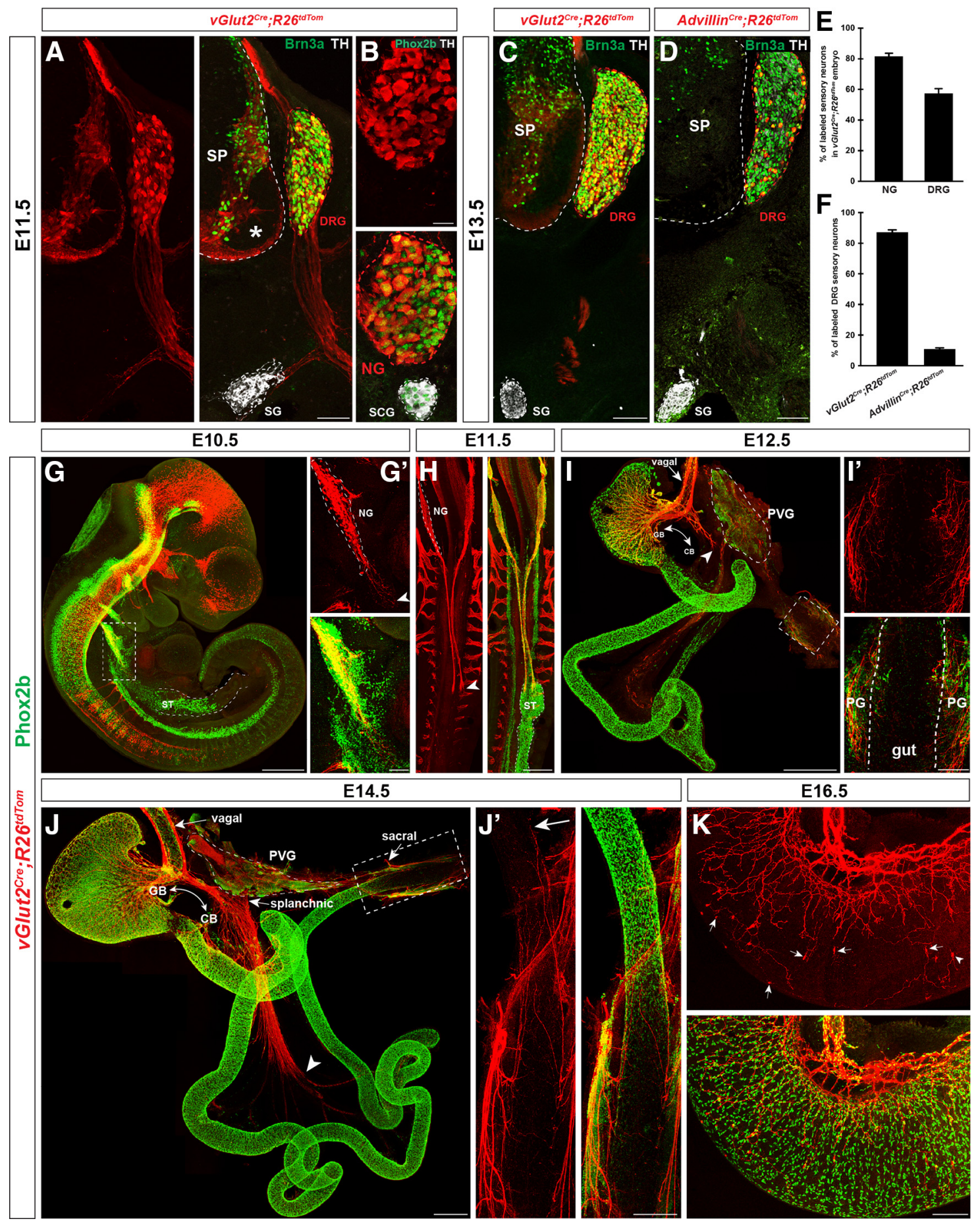

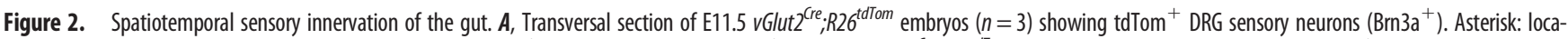
tion of motor neurons. $\boldsymbol{B}$, Representative image showing tdTom ${ }^{+}$neurons in the NG (Phox $\left.2 b^{+}\right)$of E11.5 $v \mathrm{VGlut}^{\text {(re }}, R 26^{\text {tdTom }}$ embryos $(n=3)$. Sympathetic neurons (TH $\left.{ }^{+}\right)$in the SG $(\boldsymbol{A})$ and SCG $(\boldsymbol{B})$ are not labeled by tdTom. $\boldsymbol{C}, \boldsymbol{D}$, Transversal sections showing different expression patterns of tdTom in the DRGs of $v G l u 2^{\text {Cre }} ; R 26^{\text {tdTom }}(n=3)$ and $A d v i l l i n^{\text {Cre }} ; R 26^{\text {tdTom }}(n=3)$ embryos. $\boldsymbol{E}$, Quantification of the percentage of labeled sensory neurons in the NGs and DRGs of E11.5 $\mathrm{VGlut2} 2^{\text {(re }} ;$; R2 $6^{\text {tdTom }}$ embryos $(n=1 / 3$ for NG, $n=3 / 3$ for DRG; number of ganglia per embryo/number of embryos). $\boldsymbol{F}$, Quantification of the percentage of labeled DRG sensory neurons in E13.5 vGlut2 $2^{\text {(re }} ; R 26^{\text {tdTom }}$ and Advillin ${ }^{\text {Cre }} ; R 26^{\text {tdTom }}$ embryos $\left(n=3 / 3\right.$ for vGlut2 ${ }^{\text {(re }} ; R 26^{\text {tdTom }}, n=3 / 3$ for Advillin ${ }^{\text {(re }} ; R 26^{\text {tdTom }}$; number of DRGs per embryo/number of embryos). $\mathbf{G}, \mathbf{G}^{\prime}$, Representative images showing that NG-arising vagal sensory axons project toward the developing gut in E10.5 VGlut2 $2^{\text {(re }} ; R 26^{\text {tdTom }}$ embryos $(n=3)$. The ENS is visualized by Phox $2 b$ staining. Arrowheads indicate pioneer sensory axons $(\boldsymbol{G})$. $\boldsymbol{H}$, Representative images showing that vagal sensory axons project into the stomach of E11.5 embryos (arrowhead; $n=3$ ). I, Whole-mount preparation of E12.5 guts $(n=3)$. Vagal sensory axons bifurcate into the gastric branch (GB) and celiac branch (CB). Arrowhead indicates pioneer sensory axons. Note the presence of non-neuronal tdTom ${ }^{+}$cells within the gut mesentery. $I^{\prime}$, Magnified view of the boxed area in $I$ showing that sensory axons extend into the hindgut via the PG. J, Whole-mount gut preparation of E14.5 embryos $(n=3)$ showing three sources of sensory axons (arrows). Arrowhead indicates pioneer sensory axons within the gut mesentery. $\boldsymbol{J}^{\prime}$, Magnified view of the distal hindgut showing sacral spinal sensory axons within the hindgut. Arrow indicates pioneer sensory axons. $\boldsymbol{K}$, Representative midgut segment of E16.5 embryos ( $(n=3)$. Arrows indicate enteric tdTom ${ }^{+}$neurons. Scale bars: $100 \mu \mathrm{m}\left(\boldsymbol{A}, \boldsymbol{C}, \boldsymbol{D}, \boldsymbol{I}^{\prime}, \boldsymbol{J}^{\prime}, \boldsymbol{K}\right), 25 \mu \mathrm{m}(\boldsymbol{B}), 500 \mu \mathrm{m}(\boldsymbol{G}), 50 \mu \mathrm{m}(\boldsymbol{G}), 400 \mu \mathrm{m}(\boldsymbol{H}-\boldsymbol{J})$. SP, spinal cord; SG, sympathetic ganglion; NG, nodose ganglion; ST, stomach; SCG; superior cervical ganglion; DRG, dorsal root ganglion; PVG, prevertebral ganglion; PG, pelvic ganglion. 

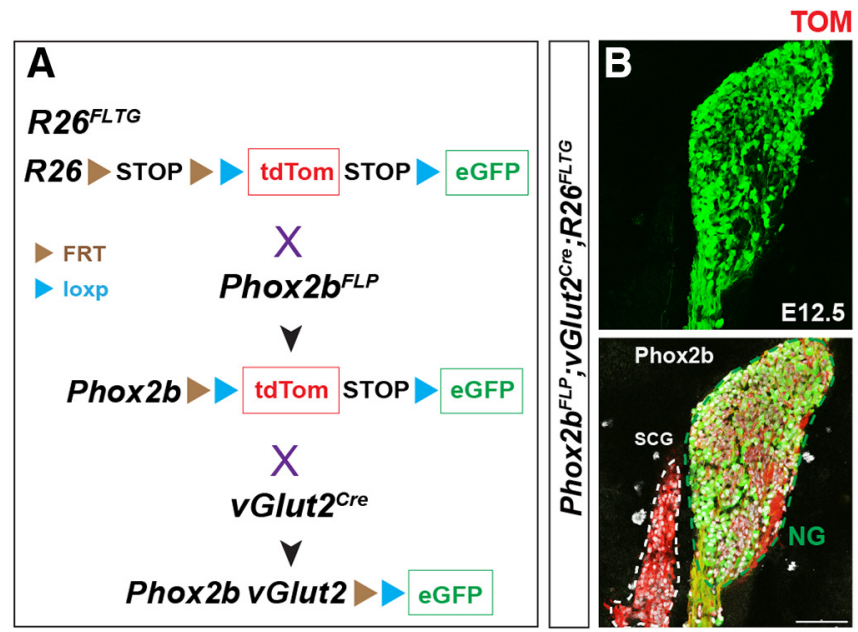

TOM GFP
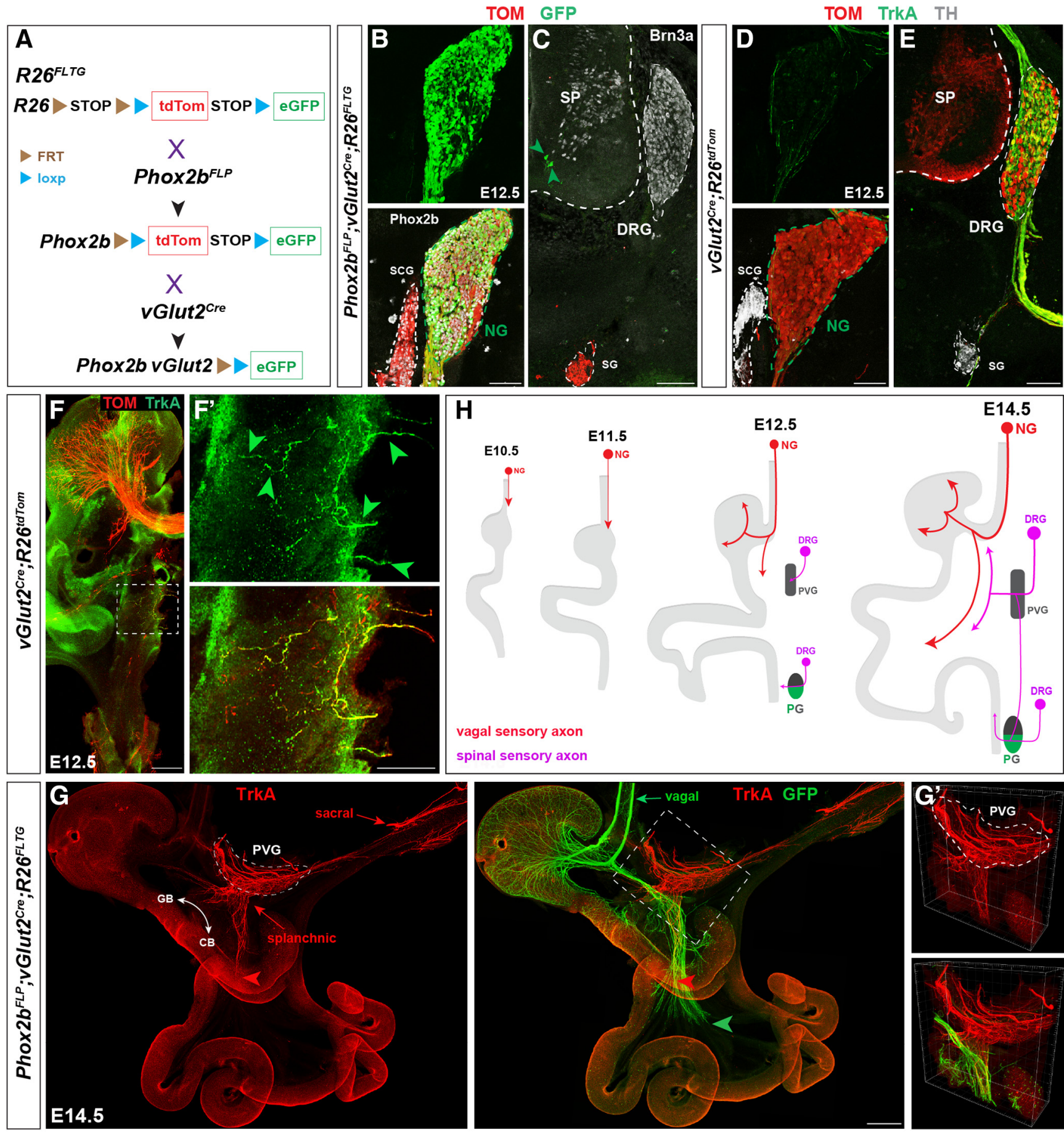

Figure 3. Distinctively marked vagal and spinal sensory trajectories. $\boldsymbol{A}$, Schematic representation of generating Phox $2 b^{F L P} ;, v G l u t 2^{\text {Cre }} ; R 26^{F L I G}$ mouse. B, $\boldsymbol{C}$, Transversal sections of E12.5 Phox $2 b^{F L P} ; v G l u t 2^{\text {ree }} ; R 26^{F L T G}$ embryos $(n=3)$ showing the expression of GFP in NG, but not in DRG, sensory neurons. Note the strong tdTom expression in SCG and SG sympathetic neurons.

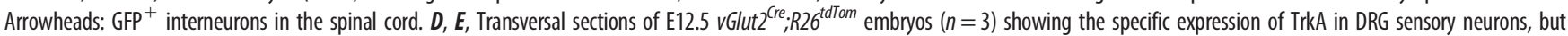
not in NG sensory and sympathetic neurons. $\boldsymbol{F}$, Representative image of E12.5 $\mathrm{VGlut}{ }^{\text {Cre }}, R 26^{\text {tdTom }}$ guts $(n=3)$ with TrkA staining. $\boldsymbol{F}^{\prime}$, Higher magnification of the boxed area in $\boldsymbol{F}$ showing TrkA ${ }^{+}$ spinal sensory axons within the PVG (arrowheads). G, Whole-mount gut preparation from E14.5 Phox2 $b^{F L P} ;$, GGlut2 ${ }^{\text {Cre }} ; R 26^{F L I G}$ embryos $(n=3)$ showing the distinctive projection patterns of vagal $\left(\mathrm{GFP}^{+}\right)$and spinal $\left(\mathrm{TrkA}^{+}\right.$) sensory axons. Red and green arrowheads indicate pioneer spinal and vagal sensory axons, respectively. $\mathbf{G}^{\prime}, 3 \mathrm{D}$ reconstruction of the boxed area in $\mathbf{G}$. $\boldsymbol{H}$, Schematic summary of the development of extrinsic afferent innervation to the gut. Scale bars: $100 \mu \mathrm{m}(\boldsymbol{B}-\boldsymbol{E}, \boldsymbol{F}), 200 \mu \mathrm{m}(\boldsymbol{F}), 400 \mu \mathrm{m}(\boldsymbol{G})$. SP, spinal cord; SG, sympathetic ganglion; NG, nodose ganglion; SCG, superior cervical ganglion; DRG, dorsal root ganglion; PVG, prevertebral ganglion; GB, gastric branch; CB, celiac branch.

Simultaneous visualization and tracing three extrinsic axonal types

Parasympathetic innervation of the GI tract is provided by both DMN-arising preganglionic parasympathetic (pre-PA) and PGarising postganglionic parasympathetic (post-PA) axons (Langley,
1921). We first sought to trace cholinergic pre-PA and post-PA neurons with the Chat ${ }^{\mathrm{Cre}} ; \mathrm{R}^{2} 6^{\text {tdTom }}$;Chat ${ }^{\mathrm{GFP}}$ mouse line. Unexpectedly, in addition to $\mathrm{GFP}^{+} / \mathrm{tdTom}^{+}$neurons in the DMN, we detected a subpopulation of $\mathrm{GFP}^{+} / \mathrm{tdTom}^{+}$sensory neurons in the NGs of E10.5 and E12.5 embryos (Fig. 5A,B). Meanwhile, tdTom 

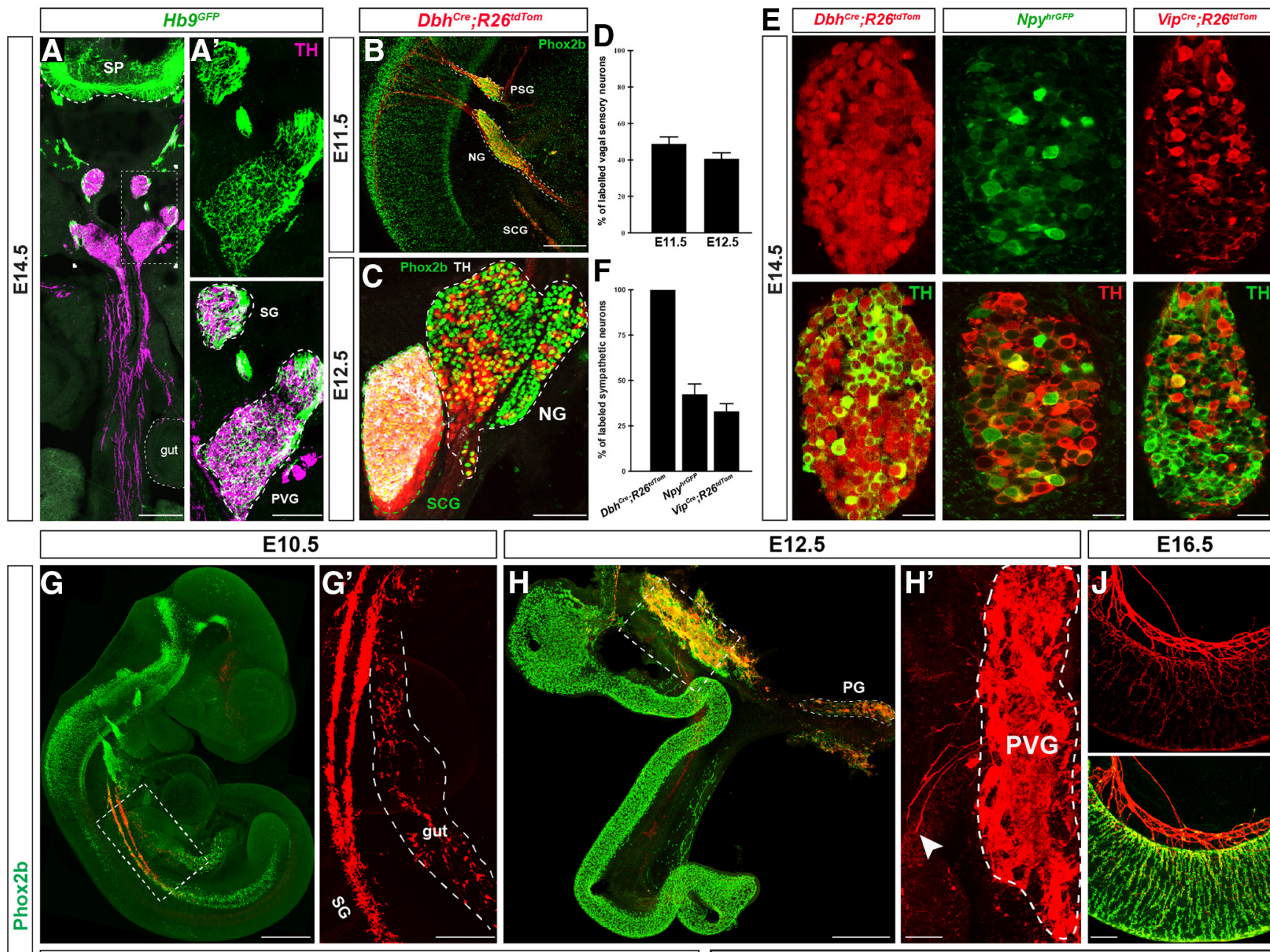

E16.5
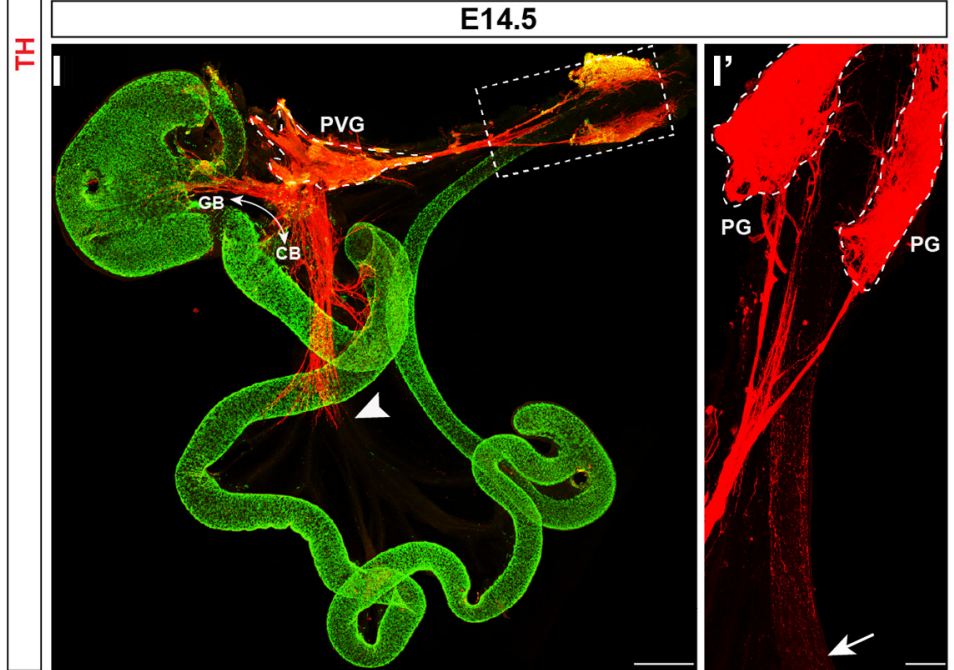

K

E12.5

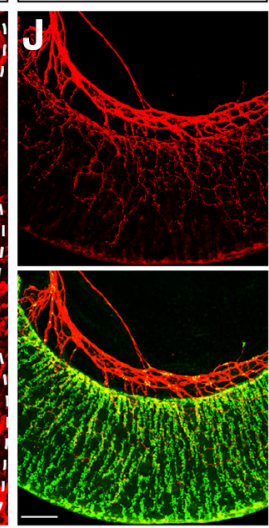

\section{E14.5}

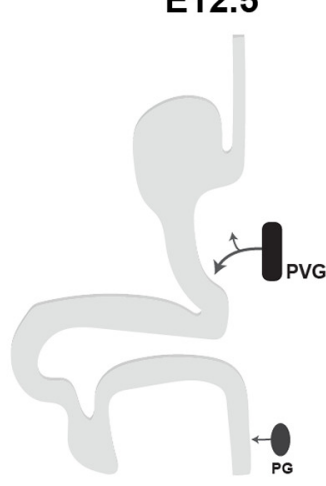

sympathetic axon

Figure 4. Spatiotemporal sympathetic innervation to the developing gut. $A, A^{\prime}$, Transversal section of E14.5 Hb $9^{G F P}$ embryos $(n=3)$. PVG neurons (TH $\left.{ }^{+}\right)$receive synaptic input from preganglionic motor neurons $\left(\mathrm{GFP}^{+}\right)$and send axons to the gut. $\boldsymbol{B}$, Lateral view of E11.5 Dbh ${ }^{\text {(re }} ; R 26^{\text {tdTom }}$ embryos $(n=3)$ showing tdTom ${ }^{+}$NG sensory neurons. Note that tdTom ${ }^{+}$neurons in the PSG. C, Transversal section of E12.5 Dbh ${ }^{\text {(re }} ; R 26^{\text {tdTom }}$ embryos $(n=3)$ showing Tom ${ }^{+} /$Phox $2 b^{+}$NG sensory neurons. TH immunoreactivities indicate the location of the SCG. $\boldsymbol{D}$, Quantification of the percentage of tdTom ${ }^{+} \mathrm{NG}$ sensory neurons in $D b h^{\text {(re }} ; R 26^{\text {tdTom }}$ embryos ( $n=6 / 3$ for both groups; number of ganglia/number of embryos). $\boldsymbol{E}$, Transversal section of the $S G$ showing the label-

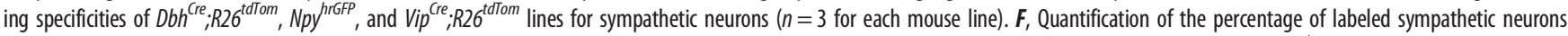
( $n=3$ for each mouse line). $\mathbf{G}, \boldsymbol{G}^{\prime}$, Representative image of E10.5 embryos $(n=3)$ with TH and Phox2b immunostaining showing scattered distribution of $\mathrm{TH}^{+}$neurons along the gut. $\boldsymbol{H}, \boldsymbol{H}^{\prime}$, Whole-mount preparation of E12.5 guts $(n=3)$. Arrowhead indicates PVG-arising pioneer axons. $I, I^{\prime}$, Whole-mount preparation of E14.5 guts $(n=3)$. PVG sympathetic axons bifurcate into gastric branch (GB) and celiac branch (CB). Arrowhead indicates pioneer sympathetic axons. Arrow indicates pioneer PG-arising sympathetic axons within the hindgut. J, Representative midgut segment of E16.5 embryos $(n=3)$. $\boldsymbol{K}$, Schematic summary of the development of gut-innervating sympathetic axons. Scale bars: $200 \mu \mathrm{m}(\boldsymbol{A}, \boldsymbol{B}, \boldsymbol{G}), 100 \mu \mathrm{m}\left(\boldsymbol{A}^{\prime}, \boldsymbol{C}, \boldsymbol{H}^{\prime}, \boldsymbol{J}, \boldsymbol{I}\right), 25 \mu \mathrm{m}(\boldsymbol{E})$, $500 \mu \mathrm{m}(G), 400 \mu \mathrm{m}(\boldsymbol{H}, I)$. SP, spinal cord; PVG, prevertebral ganglion; NG, nodose ganglion; PSG, petrosal ganglion; SCG, superior cervical ganglion; SG, sympathetic ganglion; PG, pelvic ganglion. 


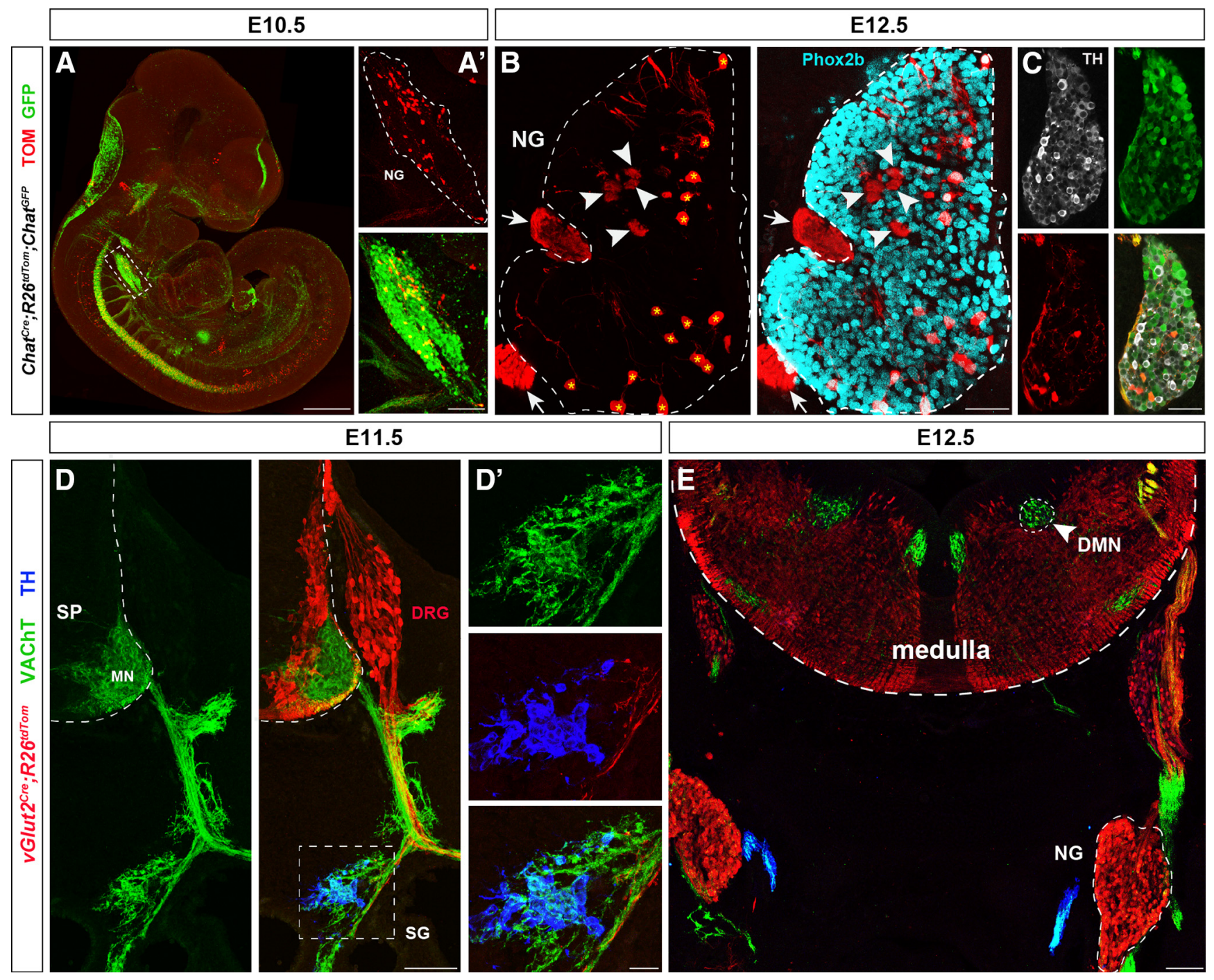

Figure 5. Characterization of intrinsic markers for parasympathetic neurons. $A, A^{\prime}$, Representative images showing both Tom ${ }^{+}$and GFP ${ }^{+}$neurons in the NG of E10.5 Chat ${ }^{\text {Cre }} ; R_{2} 6^{\text {tdTom }} ;$ Chat $^{G F P}$ embryos $(n=3)$. B, Transversal section of the NG from E12.5 Chat ${ }^{\text {(re }}, R 26^{\text {tdTom }}$ embryos $(n=3)$. Arrowheads: tdTom ${ }^{+}$axons within the NG. Arrows: NG-bypassing tdTom ${ }^{+}$axons. Asterisks:

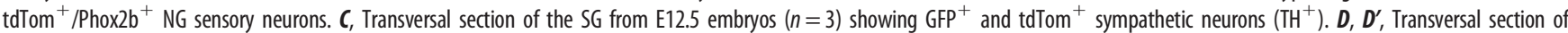
E11.5 VGlut $2^{\text {(re }} ; R 26^{\text {tdTom }}$ embryos $(n=3)$ showing the expression of VAChT in motor and sympathetic $\left(\mathrm{TH}^{+}\right)$neurons, but not in DRG sensory neurons. $\boldsymbol{E}$, Transversal section through the medulla of E12.5 embryos $(n=3)$ showing VAChT ${ }^{+}$neurons within the DMN, but not in the NG. Scale bars: $500 \mu \mathrm{m}(\boldsymbol{A}), 100 \mu \mathrm{m}\left(\boldsymbol{A}^{\prime}, \boldsymbol{D}, \boldsymbol{E}\right), 25 \mu \mathrm{m}(\boldsymbol{B}, \boldsymbol{D}), 50 \mu \mathrm{m}(\boldsymbol{C})$. NG, nodose ganglion; SG, sympathetic ganglion; SP, spinal cord; DRG, dorsal root ganglion; MN, motor neuron; DMN, dorsal motor nucleus of the vagus nerve.

and GFP were also expressed by different subpopulations of sympathetic neurons in the sympathetic chain (Fig. 5C). The vesicular acetylcholine transporter (VAChT) has also been commonly used to label cholinergic neurons (Arvidsson et al., 1997). VAChT was expressed by pre-PA neurons in the DMN, as well as by sympathetic cholinergic neurons in the PVG but not by vagal and spinal sensory neurons (Fig. 5D,E). Therefore, as demonstrated above, expression of $v$ Glut $2^{\text {Cre }} ; R 26^{\text {tdTom }}$ transgene can be used to localize sensory axons, and the combination of TH and VAChT immunoreactivities in the same transgenic embryo would allow us to simultaneously trace pre-PA and post-PA axons $\left(\mathrm{tdTom}^{-} / \mathrm{TH}^{-} / \mathrm{VAChT}^{+}\right)$, sympathetic $\left(\mathrm{tdTom}^{-} / \mathrm{TH}^{+} / \mathrm{VAChT}^{+}\right)$, and visceral sensory $\left(\operatorname{tdTom}{ }^{+} / \mathrm{TH}^{-} / \mathrm{VAChT}^{-}\right)$axons.

At E10.5, axons from scattered pre-PA $\mathrm{VAChT}^{+}$neurons fasciculated and extended from the hindbrain. Most pre-PA axons bypassed, with a minority of axons projecting through (Fig. $5 B$ ), the NG to extend along pioneer sensory axons (Fig. $6 A, A^{\prime}$ ). PrePA axons followed sensory axons along the esophagus and entered the stomach at E11.5 (Fig. $6 B, B^{\prime}$ ). By E12.5, both sensory and pre-PA axons bifurcated into gastric and celiac trajectories (Fig. 6C). Close observations revealed that pre-PA axons invariably projected along with, but slightly behind of, sensory afferents within the stomach (Fig. $6 C^{\prime}, D$ ). Meanwhile, PVG sympathetic neurons started to send out their projections to the GI tract to join pre-PA and vagal sensory axons (Fig. $6 C^{\prime \prime}$ ). Then, sympathetic axons also split into two trajectories: gastric branch along the preformed sensory and parasympathetic trajectories to the stomach, and celiac branch where three types of axons kept close association with each other in the gut mesentery at E14.5 (Fig. $6 E)$. Notably, the most advanced axons along the celiac trajectory changed from sympathetic fibers at E12.5 to sensory or pre-PA fibers at E14.5 (Fig. $6 E^{\prime}$ ), indicating that various types of axons might possess different axonal extension rate during development.

By E14.5, the gut has been fully innervated by enteric $\mathrm{VAChT}^{+}$neurons, thus hampering us to trace PG-arising postPA axons into the hindgut with VAChT immunoreactivity. By 

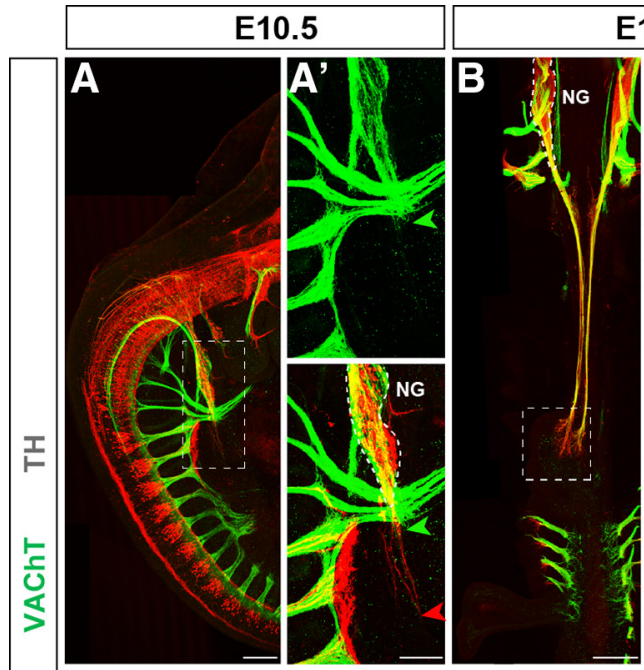

E11.5

$\mathrm{E} 12.5$
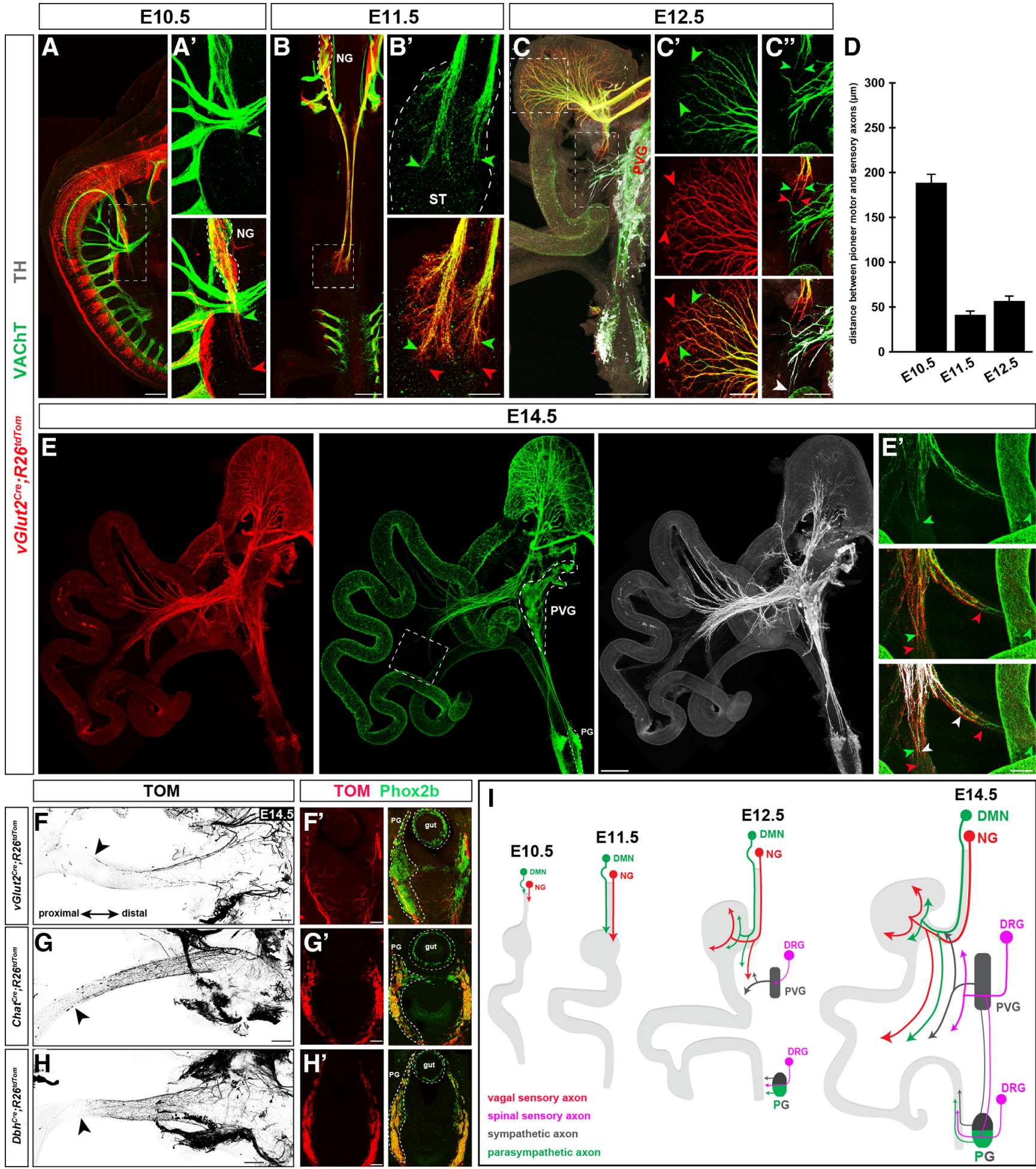

parasympathetic axon

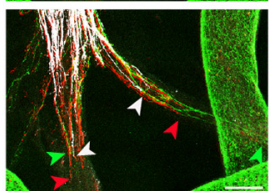

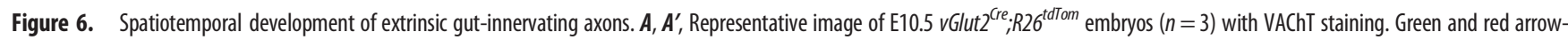
heads indicate pioneer pre-PA and sensory axons, respectively. $\boldsymbol{B}, \boldsymbol{B}^{\prime}$, Whole-mount view of E11.5 embryos $(n=3)$ with ventral visceral organs being removed. Magnified view of the boxed area $(\boldsymbol{B})$ showing delayed pre-PA axons (green arrowheads) compared with sensory axons (red arrowheads) within the stomach. $C$, Whole-mount preparation of E12.5 vGlut2 ${ }^{\text {(ree }} ; R 26^{\text {tdTom }}$ guts ( $\left.n=3\right)$. $C^{\prime}$, Higher magnification of the boxed area within the stomach showing delayed extension of pre-PA axons. Green and red arrowheads indicate pioneer pre-PA axons and sensory axons, respectively. $C^{\prime \prime}$, Higher magnification of another boxed area showing pre-PA celiac axons (green arrowhead) catch up with the advancing sensory axons (red arrowhead) to contact with sympathetic axons from the PVG (white arrowhead). D, Quantification of the distance between pioneer sensory axons and pre-PA axons ( $n=4,3$, and 3 for E10.5, E11.5, and E12.5 embryos, respectively). $\boldsymbol{E}$, Whole-mount view of the GI tract from E14.5 embryos $(n=3)$. $\boldsymbol{E}^{\prime}$, Higher magnification of the boxed area showing the intimate association of distinctive axons within the gut mesentery. Green, white, and red arrowheads indicate pioneer pre-PA, sympathetic, and sensory axons. $\boldsymbol{F}-\boldsymbol{H}$, Distal hindguts from E14.5 VGlut $2^{\text {Cre }} ; R 26^{\text {tdTom }}$, Chat ${ }^{\text {Cre }} ; R 26^{\text {tdTom }}$, and Dbh ${ }^{\text {Cre }} ; R 26^{\text {tdTom }}$ embryos showing extrinsic sensory,

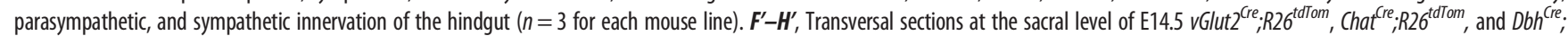
$R 26^{\text {tdTom }}$ embryos with Phox2b staining showing the Chat tom ${ }^{\text {tdo }}$ and $D b h^{\text {tdTom }}+$, but not $v G l u t 2^{\text {tdTom }+}$, neurons within the PG ( $n=3$ for each mouse line). Note that DRG sensory axons extend through the PG to innervate the hindgut $(\boldsymbol{F})$. I, Schematic summary of the extrinsic innervation of the gut from E10.5 to E14.5. Scale bars: $200 \mu \mathrm{m}(\boldsymbol{A}), 100 \mu \mathrm{m}\left(\boldsymbol{A}^{\prime}, \boldsymbol{B}^{\prime}, \boldsymbol{C}^{\prime}, \boldsymbol{C}^{\prime \prime}, \boldsymbol{E}^{\prime}, \boldsymbol{F}-\boldsymbol{H}, \boldsymbol{F}^{\prime}-\boldsymbol{H}\right)$, $400 \mu \mathrm{m}(\boldsymbol{B}, \boldsymbol{C}, \boldsymbol{E})$. DMN, dorsal motor nucleus of the vagus nerve; NG, nodose ganglion; DRG, dorsal root ganglion; ST, stomach; PVG, prevertebral ganglion; PG, pelvic ganglion. 
Table 1. Specificity of mouse lines and antibodies for various types of neurons

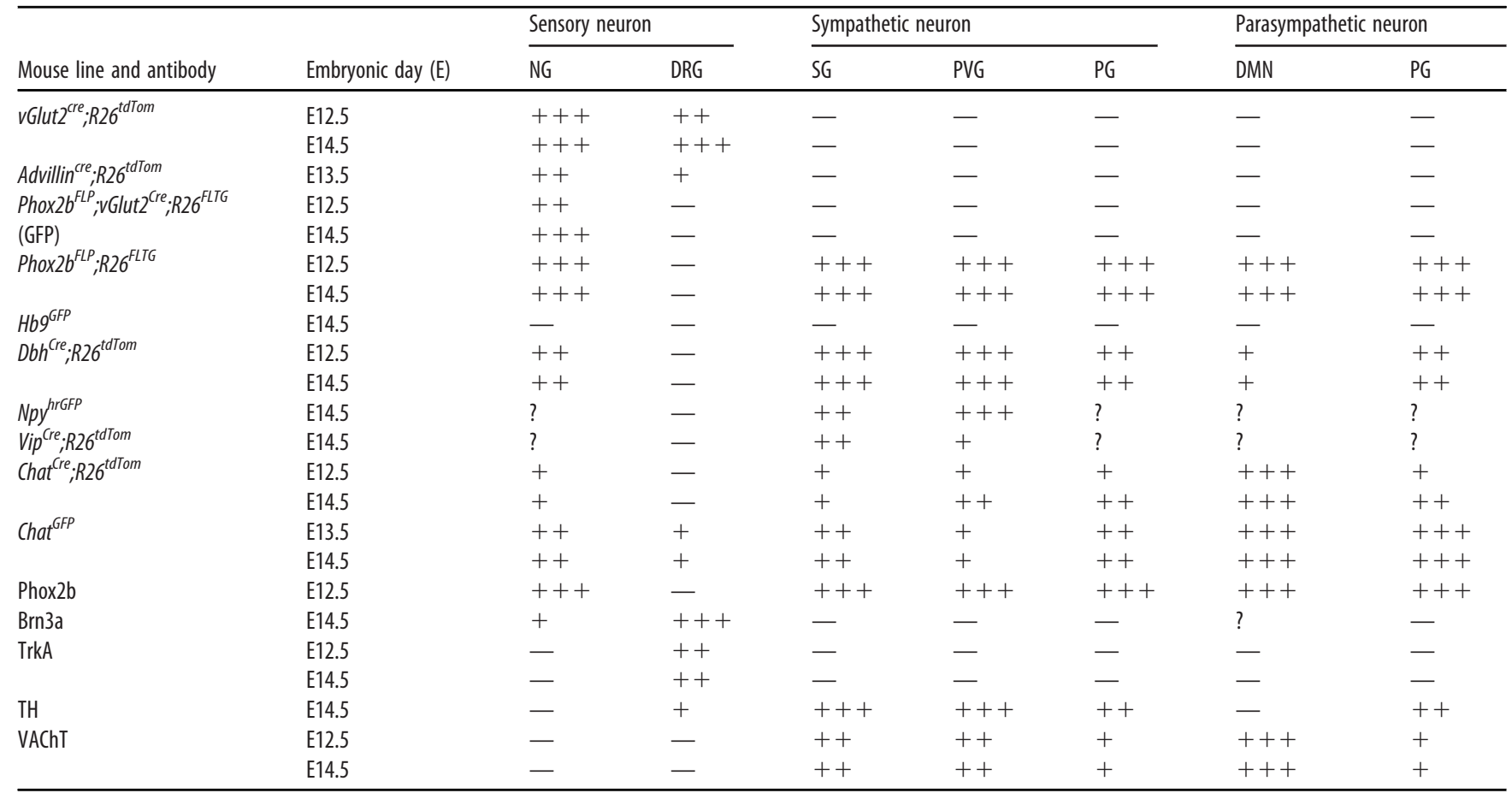

-, no neurons being labeled; + , scattered neurons being labeled; ++ , most neurons being labeled; +++ , all neurons being labeled; ?, not yet examined.

NG, nodose ganglion; DRG, dorsal root ganglion; SG, sympathetic ganglion; PVG, prevertebral ganglion; PG, pelvic ganglion; DMN, dorsal motor nucleus of the vagus nerve.

contrast, in the E14.5 gut from Chat ${ }^{\mathrm{Cre}} ; \mathrm{R}_{2} 6^{\text {tdTom }}$ embryos, enteric Chat ${ }^{\text {tdTom }}{ }^{+}$neurons occurred in a rostral-to-caudal manner, and only a few Chat ${ }^{\text {tdTom }}{ }^{+}$were detected within the hindgut, therefore making it possible to distinguish post-PA cholinergic axons from enteric cholinergic axons within the distal hindgut (Fig. $6 G)$. Given that DRG sensory neurons were not genetically labeled in $D b h^{\text {Cre }} ; R 26^{\text {tdTom }}$ and Chat ${ }^{\text {Cre }} ; R 26^{\text {tdTom }}$ embryos, we therefore employed $v$ Glut $2^{C r e} ; R 26^{\text {tdTom }}$, Chat ${ }^{C r e} ; R 26^{\text {tdTom }}$, and $D b h^{C r e} ; R 26^{\text {tdTom }}$ lines to trace DRG sensory, pelvic post-PA, and pelvic sympathetic axons into the hindgut, respectively. In line with the classic notion that the PG consists of mixed cholinergic and noradrenergic neurons for parasympathetic and sympathetic innervations of the lower GI tract (Keast, 1999), Chat ${ }^{\text {tdTom }+}$ and $\mathrm{Dbh}^{\text {tdTom }+}$ neurons, but not vGlut2 $2^{\text {tdTom }+}$ neurons, were detected within the PGs of E14.5 embryos (Fig. $6 F^{\prime}-H^{\prime}$ ). These three types of extrinsic axons demonstrated the same distal-toproximal projection directions within the hindgut (Fig. $6 F-H)$.

In summary, with characterized intrinsic markers targeting vagal sensory, spinal sensory, sympathetic, and parasympathetic axons, respectively (Table 1), we revealed that extrinsic innervation of the GI tract spatiotemporally develops in the following sequence (Fig. 6I). First, NG sensory axons extend caudally along the esophagus at E10.5. Second, with a short delay, pre-PA axons emanate from the DMN and follow the preexisting sensory axons to enter the stomach at E11.5. Third, at E12.5, sympathetic axons from the PVG initiate their projections to the gut to establish trajectories for both vagal and spinal sensory nerves. Fourth, between E12.5 and E14.5, vagal sensory, spinal sensory, sympathetic, and parasympathetic axons extend in close association along the gut mesentery toward the GI tract. Simultaneously, spinal sensory, sympathetic, and parasympathetic axons extend into the hindgut either from or via the PG and innervate the hindgut in a distal-to-proximal direction.
Distinctive extrinsic axonal trajectories within the GI tract

Next, we traced extrinsic axons within the GI tract. At E16.5, the presence of a large amount of enteric cholinergic neurons impeded us to trace pre-PA and post-PA axons within the gut. By contrast, the presence of a few scattered $\operatorname{tdTom}^{+}$neurons along the E16.5 vGlut2 ${ }^{\mathrm{Cre}} ; \mathrm{R}^{\text {tdTom }}{ }^{\text {tTo }}$ gut allowed us to follow sensory axons into the gut (Fig. $2 K$ ). Therefore, we performed $\mathrm{TH}$ staining on the GI tract from E16.5 v Glut ${ }^{\text {Cre }} ; R_{2} 6^{\text {tdTom }}$;Chat ${ }^{\text {GFP }}$ embryos to simultaneously visualize extrinsic sensory $\left(\operatorname{tdTom}^{+}\right)$, sympathetic $\left(\mathrm{TH}^{+}\right)$axons, and enteric neurons $\left(\mathrm{GFP}^{+}\right)$within the gut. In contrast to intimately associating within the gut mesentery, sensory and sympathetic axons demonstrated distinctive projection patterns within the gut wall (Fig. $7 A, A^{\prime}$ ). Transversal sections of E16.5 midgut revealed that both sensory and sympathetic axons extended mainly along the myenteric plexus, but not along the arteries, within the gut (Fig. $7 B$ ). In summary, sensory and sympathetic axons project in close association along the gut mesentery and innervate the gut with distinctive projection patterns.

\section{Extension of pre-PA axons depended on preexisting sensory,} but not sympathetic, trajectories

The close association of vagal sensory and vagal pre-PA axons raises the possibility that whether, like their epibranchial counterparts (Coppola et al., 2010), sensory axons also serve as a scaffold for later-extending pre-PA axons. To test this idea, we established $v$ Glut ${ }^{\text {Cre }} ; R 26^{\text {tdTom }} ; R 26^{\text {DTA }}(\Delta S N)$ mice to specifically express diphtheria toxin (DTA) in $v$ Glut $2^{+}$neurons to eliminate sensory neurons. Quantitative analyses revealed that appropriate $70 \%$ of NG sensory neurons were ablated in E12.5 $\Delta S N$ embryos (Fig. $8 A, B$ ). Subsequently, we examined $\mathrm{DMN}$-arising pre-PA axonal extension in the absence of vagal sensory pathways. At E11.5, the extension of pre-PA axons in $\triangle S N$ embryos was 


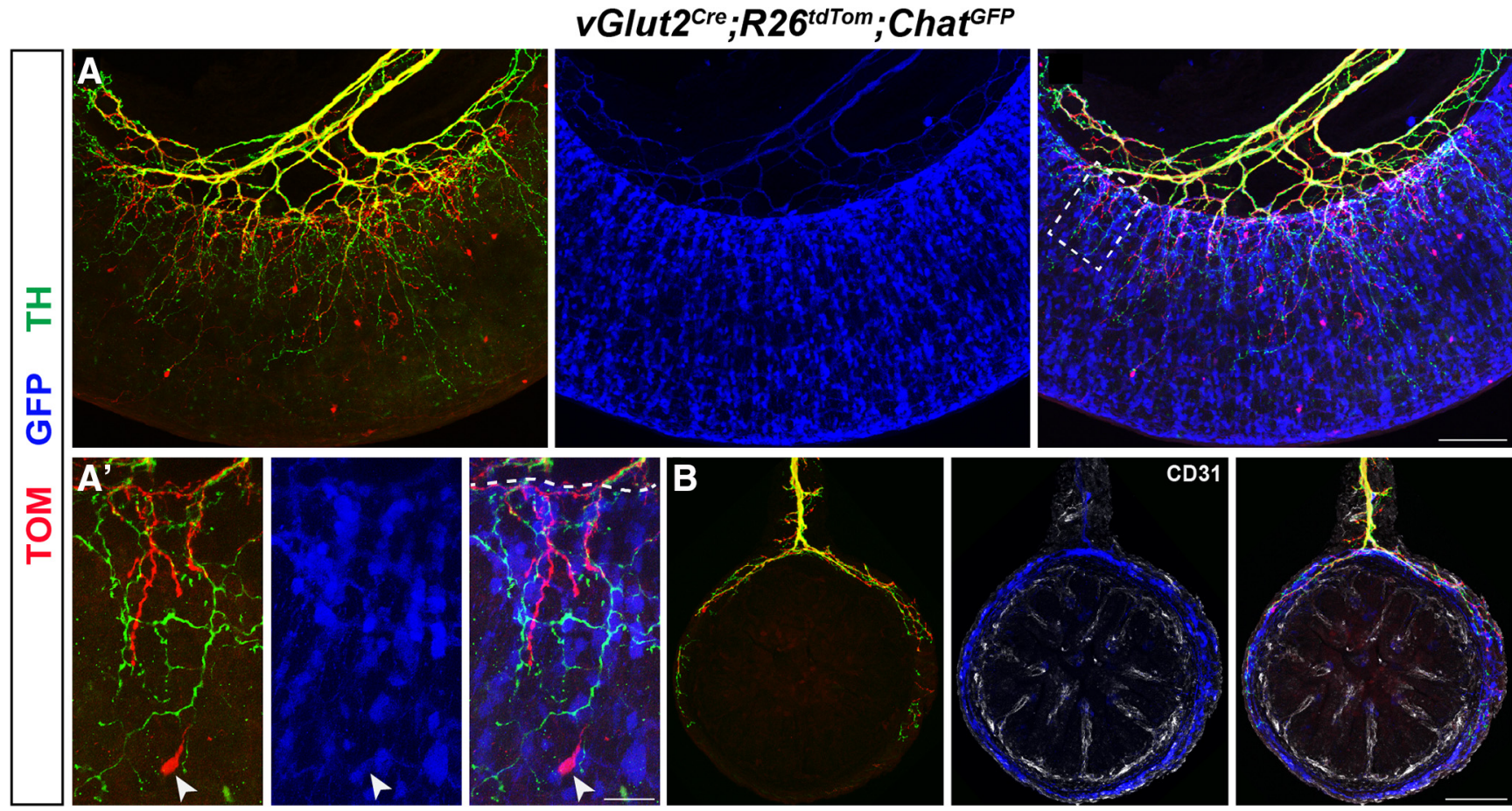

Figure 7. Distinctive projection patterns of sensory and sympathetic axons within the E16.5 gut. $A$, Representative midgut segment from E16.5 vGlut ${ }^{\text {Cre }} ; R 26^{\text {tdTom }} ; C$ hat ${ }^{G F P}$ embryos $(n=3)$ showing sympathetic $\left(\mathrm{TH}^{+}\right)$and sensory (tdTom ${ }^{+}$) axons within the gut. $\boldsymbol{A}^{\prime}$, Magnified view of the boxed area demonstrating distinctive projection patterns of sensory and sympathetic axons within the gut. Arrowheads indicate one enteric Tom ${ }^{+} / \mathrm{GFP}^{+}$neuron. $\boldsymbol{B}$, Transversal section of E16.5 midguts $(n=3)$ showing that both sensory $\left(\right.$ tdTom $\left.^{+}\right)$and sympathetic $\left(\mathrm{TH}^{+}\right)$axons project mainly along the myenteric plexus $\left(\mathrm{GFP}^{+}\right)$, but not along the arteries $\left(\mathrm{CD}^{+} 1^{+}\right)$. Scale bars: $100 \mu \mathrm{m}(\boldsymbol{A}), 50 \mu \mathrm{m}(\boldsymbol{A}), 100 \mu \mathrm{m}(\boldsymbol{B})$.

dramatically delayed compared with that in Control embryos, with most advanced axons still in the middle of the esophagus (Fig. 8C). Moreover, pre-PA axonal bundles on both sides became significantly defasciculated, and some axons projected diffusely across the midline to merge with the axons on the other side (Fig. $8 C, D$ ). One day later at E12.5, while the stomach of Control embryos was heavily innervated, most of the stomach of $\triangle S N$ embryos was devoid of innervation, with pre-PA axons stalled and accumulated in the region around the cardia of the developing stomach (Fig. 8E). Upon further development at E14.5, impairment of the vagal sensory pathways continued to affect pre-PA innervation of the stomach, and many pre-PA axons stalled or broke down into axonal debris within the stomach (Fig. 8F,G). Co-detection with Phox 2 b revealed that the ENS in $\triangle S N$ embryos developed normally (Fig. $8 F^{\prime}, G^{\prime}$ ), thus excluding the possibility that the defective projection of pre-PA axons was because of the abnormal development of the ENS. Quantification of the gastric area occupied by pre-PA axons demonstrated that partial delay or ablation of visceral sensory projections seriously affected pre-PA axonal projections within the vagus nerves (Fig. $8 H$ ). Thus, we concluded that pioneer vagal sensory axons served as a scaffold for the extension of prePA axons.

We further noticed that pre-PA axons in $\triangle S N$ embryos, instead of extending coordinately with sympathetic axons through the gut mesentery as their counterparts in Control embryos (Fig. 8F, red arrowheads), exhibited highly aberrant projections toward the PVG (Fig. 8G, red arrowheads), thus implicating that ablating sensory trajectories triggered erratic projection of pre-PA axons within the gut mesentery. However, given that sympathetic axons also exhibited obvious delayed extension in $\triangle S N$ embryos (Fig. $8 F, G$, green arrowheads), defective pre-PA axonal extension might be either the direct effect of ablating sensory trajectories, or the indirect effect of erratic sympathetic axon development in $\triangle S N$ embryos. To distinguish between these two possibilities, we examined pre-PA trajectories in the absence of sympathetic fibers with the previously established $D b h^{C r e} ; R 26^{D T A}(\triangle S E)$ mouse line (Fig. 9A-C; Wang et al., 2014). Although a subpopulation of NG sensory neurons expressed Dbh during the early stage of development (Fig. 4B,C), quantitative analysis showed that most NG sensory neurons were still present in E14.5 $\triangle S E$ embryos (Fig. 9B). In contrast to the defective extension after sensory axon ablation, pre-PA axons in the gut mesentery of $\triangle S E$ embryos exhibited comparable projection patterns with those found in Control embryos, except that $\mathrm{VAChT}^{+}$axons from the PG were dramatically decreased in the hindgut, possibly resulting from the ablation of $\mathrm{Dbh}^{+} / \mathrm{VAChT}^{+}$ sympathetic axons (Fig. 9D-F). As sympathetic axons were dispensable for pre-PA axonal extension, we concluded that the erratic pre-PA axonal extension within the gut mesentery of $\Delta S N$ embryos was directly resulted from ablation of sensory trajectories. Therefore, spinal sensory axons, together with vagal sensory axons, have attractive effect on pre-PA axonal extension within the gut mesentery.

Do the sympathetic trajectories serve as a template to guide later-extending spinal sensory axons? To address this, we performed TrkA immunodetection on E14.5 GI tract from $\triangle S E$ embryos and found that spinal sensory axons demonstrated comparable projection patterns with those in Control embryos (Fig. 9G-I). Moreover, given the attractive effect of vagal sensory axons on pre-PA projections, unchanged pre-PA projection patterns in $\triangle S E$ embryos also indirectly indicated that vagal sensory axons projected in a relatively normal pattern in the absence of sympathetic trajectories. Therefore, we concluded that sympathetic axons were also dispensable for vagal sensory axonal projections. In summary, these data reveal that sympathetic axons are dispensable for both pre-PA and sensory trajectories. 

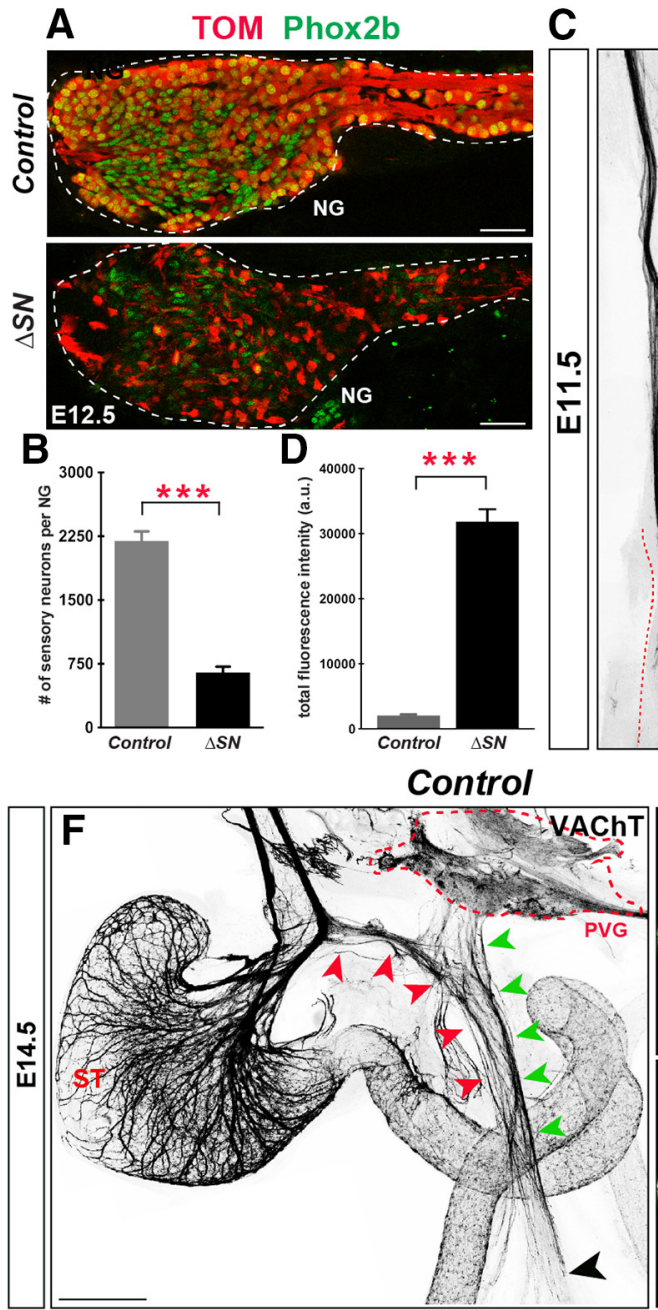

H

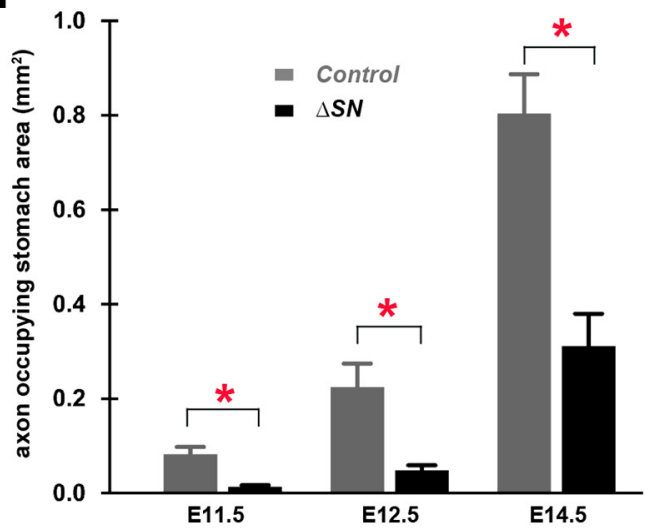

C

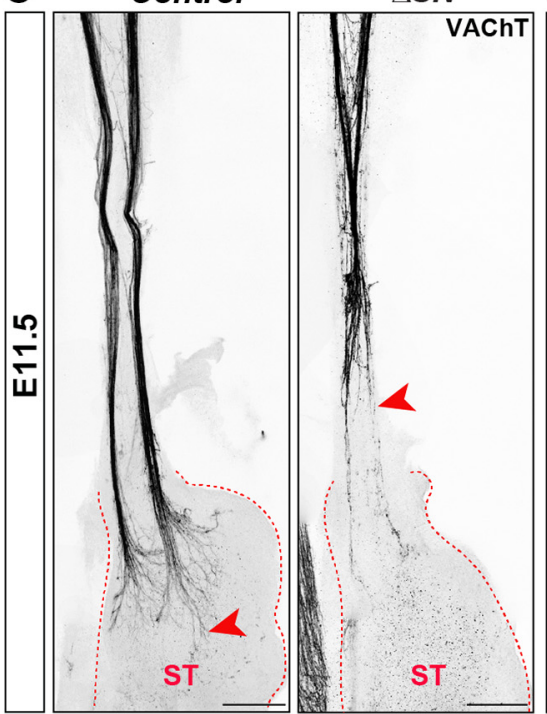

$\mathbf{E}$

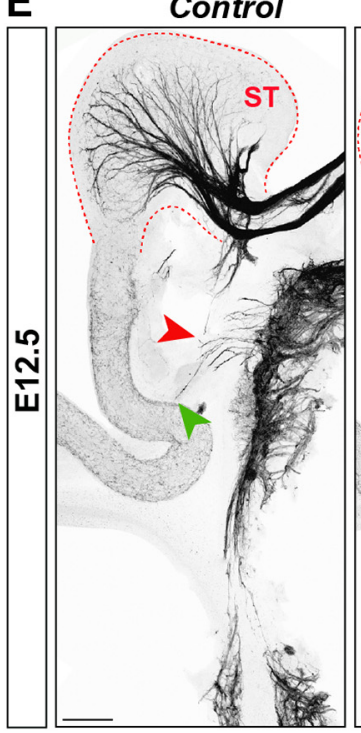

$\triangle S N$

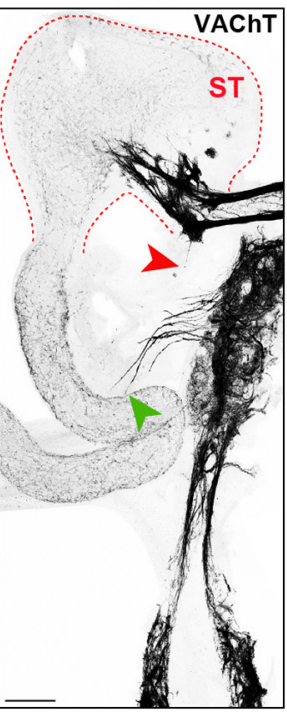

ST

$\triangle S N$
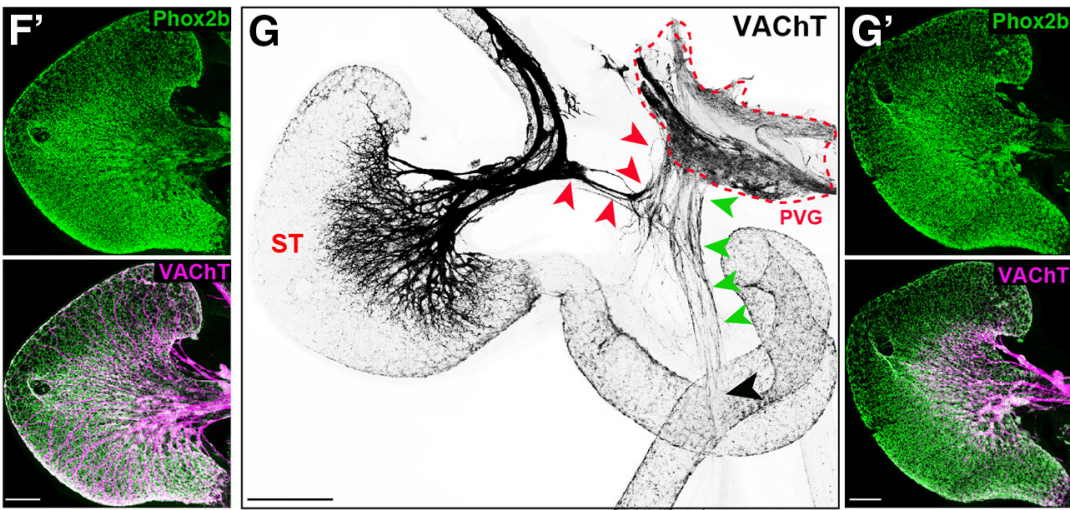

I
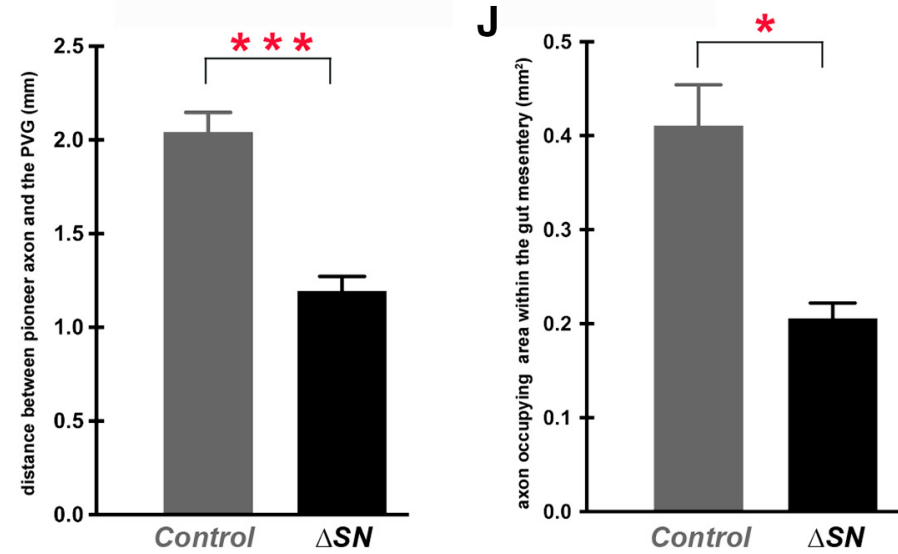

Figure 8. Erratic pre-PA axonal extension in the absence of sensory trajectories. $A$, Transversal sections from E12.5 Control (vGlut2 $\left.{ }^{\text {(re }} ; R 26^{\text {tdTom }} ; n=3\right)$ and $\Delta S N\left(v G l u t 2^{\text {(re }} ; R 26^{\text {tdTom }} ; R 26^{\text {DTA }}\right.$; $n=3$ ) embryos showing the great reduction of NG sensory neurons (tdTom ${ }^{+} / \mathrm{Phox} \mathrm{b}^{+}$) in the $\triangle S \mathrm{~N}$ embryo. $\boldsymbol{B}$, Quantification of NG sensory neurons from E12.5 Control and $\triangle S N$ embryos ( $n=6 / 3$ for Control embryo, $n=8 / 4$ for $\Delta S N$ embryo; number of ganglia/number of embryos. $t$ test, $\left.{ }^{* * *} p<0.0001\right)$. C, Ventral views of E11.5 Control $(n=3)$ and $\Delta S N(n=3)$ embryos with VAChT staining showing the obvious delay of pre-PA axons in $\triangle S N$ embryo. Arrowheads indicate pioneer pre-PA axons. $D$, Quantification of the fluorescence intensity of VAChT signal along the midline between two vagus nerve trunks $\left(n=3\right.$ for both groups; $t$ test, $\left.{ }^{* * *} p<0.0001\right)$. $\boldsymbol{E}$, Whole-mount views of E12.5 gut from Control $(n=3)$ and $\Delta S N(n=3)$ embryos. Red arrowheads: pioneer pre-PA axons; green arrowheads: PVG-arising sympathetic axons. Note normal sympathetic axon projections in $\triangle S N$ embryo. $F, G$, Whole-mount preparations of E14.5 gut from Control $(n=3)$ and $\triangle S N(n=3)$ embryos showing defective pre-PA innervation of the stomach in $\triangle S N$ embryo. Red arrowheads indicate pre-PA axons, and green arrowheads indicate PVG-arising sympathetic axons, Note the erratic projection of pre-PA axons and obvious delayed extension of sympathetic axons. $\boldsymbol{F}^{\prime}, \mathbf{G}^{\prime}$, Whole-mount views of the stomach from Control $(n=3)$ and $\Delta S N$ $(n=3)$ embryos with Phox2b and VAChT staining. $\boldsymbol{H}$, Quantification of the area occupied by pre-PA axons in the stomach of Control and $\Delta S N$ embryos $(n=3$ for all the groups; E11.5, Kruskal-Wallis test, ${ }^{*} p=0.0143$; E12.5, Kruskal-Wallis test, ${ }^{*} p=0.0261 ; \mathrm{E} 14.5, t$ test, $\left.{ }^{*} p=0.0104\right)$. I, Quantification of the distance between the PVG and the most advanced VAChT ${ }^{+}$axon within the gut mesentery $\left(n=3\right.$ for each group; Kruskal-Wallis test, $\left.{ }^{* * *} p<0.0001\right)$. J, Quantification of the occupying area of VAChT ${ }^{+}$axons within the gut mesentery $(n=3$ for each group; Kruskal-Wallis test, $\left.{ }^{*} p=0.0113\right)$. Scale bars: $50 \mu \mathrm{m}(\boldsymbol{A}), 200 \mu \mathrm{m}\left(\boldsymbol{C}, \boldsymbol{E}, \boldsymbol{F}^{\prime}, \boldsymbol{G}\right), 400 \mu \mathrm{m}(\boldsymbol{F}, \mathbf{G})$. NG, nodose ganglion; ST, stomach; PVG, prevertebral ganglion. 

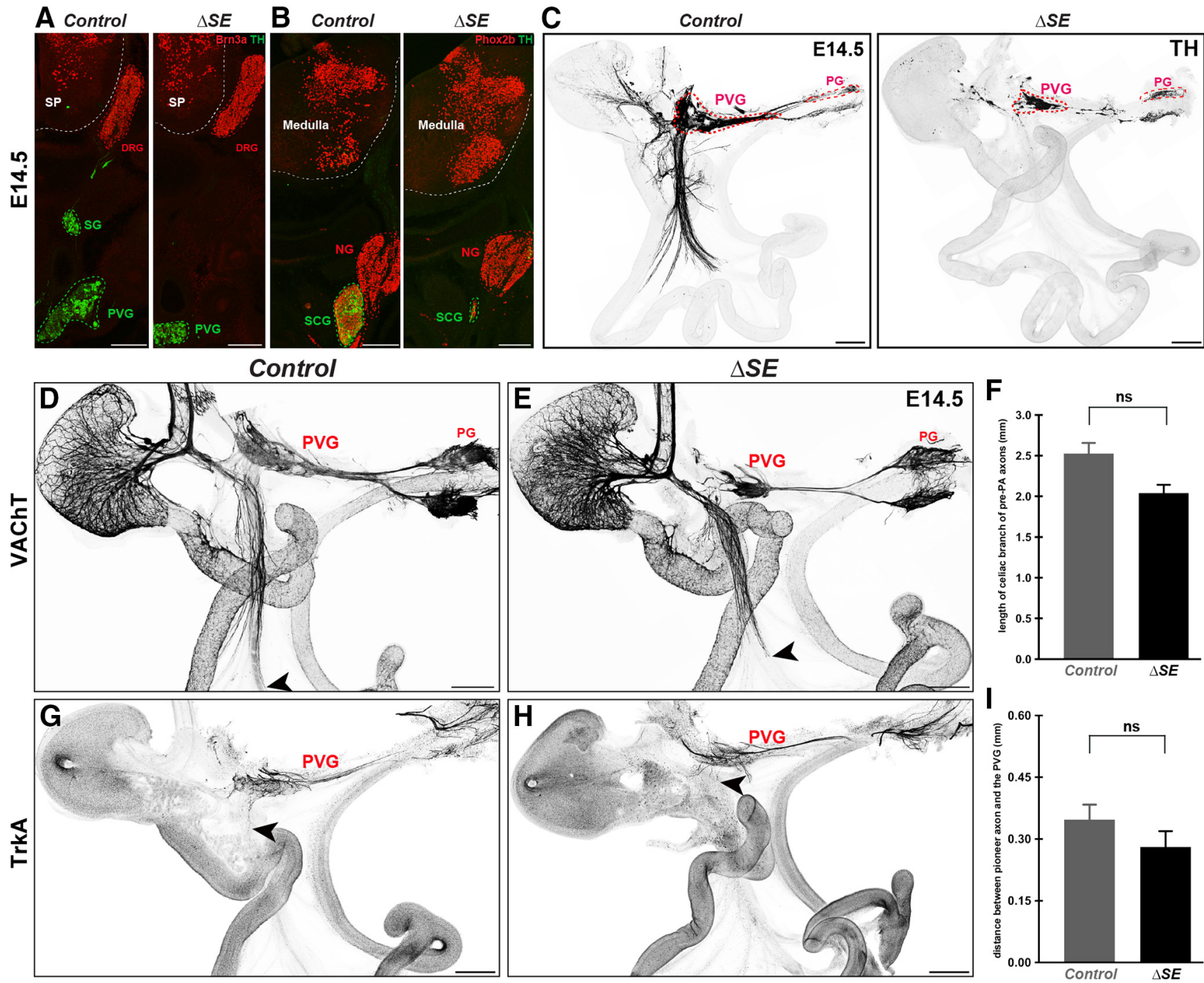

Figure 9. Pre-PA axons project independently of sympathetic fibers. $A$, Transversal sections of E14.5 Control $(n=3)$ and $\triangle S E$ (Dbh $\left.h^{\text {Cre }} ; R 26^{\text {DTA }} ; n=3\right)$ embryos showing the complete absence of SG, and the great size reduction of PVG, but normal DRGs, in $\triangle S E$ embryos. $B$, Transversal sections through the medullas of E14.5 Control $(n=3)$ and $\triangle S E(n=3)$ embryos showing the great reduction of SCG sympathetic neurons in $\triangle S E$ embryo. C, Great reduction of sympathetic axons (TH $\left.{ }^{+}\right)$in the gut mesentery of E14.5 $\triangle S E$ embryo $(n=3)$ compared with that in Control embryo $(n=3)$. $\boldsymbol{D}, \boldsymbol{E}$, Unchanged pre-PA trajectories in the absence of sympathetic pathways ( $n=3$ for each group). Arrowheads: pioneer pre-PA axons. Arrows: pioneer post-PA axons within the hindgut. Note the obvious reduction of the size of the PVG in the $\triangle S E$ embryo. $F$, Quantification of the length of VAChT ${ }^{+}$pre-PA celiac branch within the gut mesentery $(n=3$ for each group; $p=0.0615$; ns, not significant). $\mathbf{G}, \boldsymbol{H}$, Whole-mount preparations of E14.5 gut from Control $(n=3)$ and $\triangle S E(n=3)$ embryos with TrkA staining. TrkA ${ }^{+}$spinal sensory axons in $\triangle S E$ embryos project in a similar pattern as those in Control embryo. Arrowheads indicate pioneer spinal sensory axons. $I$, Quantification of the distance between most advanced TrkA ${ }^{+}$axons and the PVG ( $n=3$ for each group; $p=0.2389$; ns, not significant). Scale bars: $200 \mu \mathrm{m}(\boldsymbol{A}, \boldsymbol{B}), 400 \mu \mathrm{m}(\boldsymbol{C}-\boldsymbol{E}, \mathbf{G}, \boldsymbol{H})$. DRG, dorsal root ganglion; SG, sympathetic ganglion; SCG; superior cervical ganglion; PVG, prevertebral ganglion; NG, nodose ganglion; PG, pelvic ganglion.

\section{Sympathetic axon trajectories are configured by sensory} trajectories

Finally, we asked whether sympathetic projections are affected by sensory pathways. We first examined sympathetic extension in $\Delta S N$ embryos. As sympathetic axons extended ahead of sensory axons, the initial projection of sympathetic axons from the PVG developed normally in E12.5 $\triangle S N$ embryos (Fig. 8E, green arrowheads). However, $3 \mathrm{~d}$ later at E15.5, ablation of sensory trajectories resulted in mild, but significant, delayed projection of sympathetic axons (Fig. 10A-C). Provided that this mild erratic projection of sympathetic axons might be because of partial elimination of vagal and/or spinal sensory neurons, we co-cultured PVGs and NGs or DRGs with our previously established coculture assay to directly examine the effect of sensory axons on sympathetic axons in vitro (Wang and Marquardt, 2012). In contrast to sensory axons which extended unaffectedly by the presence of PVG explant, significantly more sympathetic axons were detected within the NG and DRG explants (Fig. 10D). We further quantified the heterotypic sympathetic-sensory axonal interactions. Most sensory growth cones ignored the presence of sympathetic axons and crossed the sympathetic axon shafts. By contrast, the majority of sympathetic growth cones were detected to intimately adhere to sensory axons to track along the preextending sensory axons (Fig. $10 E-G$ ), implicating that both vagal and spinal sensory axons effectively attract the extension of sympathetic axons, whereas sympathetic axons exert little effects on sensory extension. Together, sympathetic trajectories are attractively configured by visceral sensory axons (Fig. 11). 
A Brn3a TH B

Control
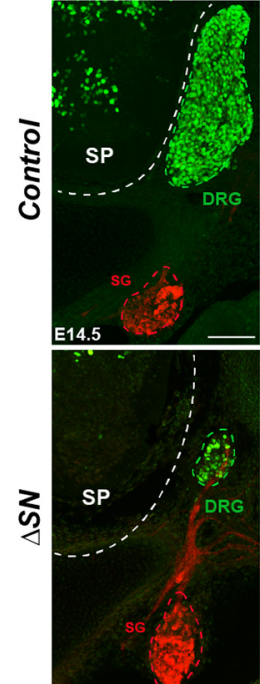

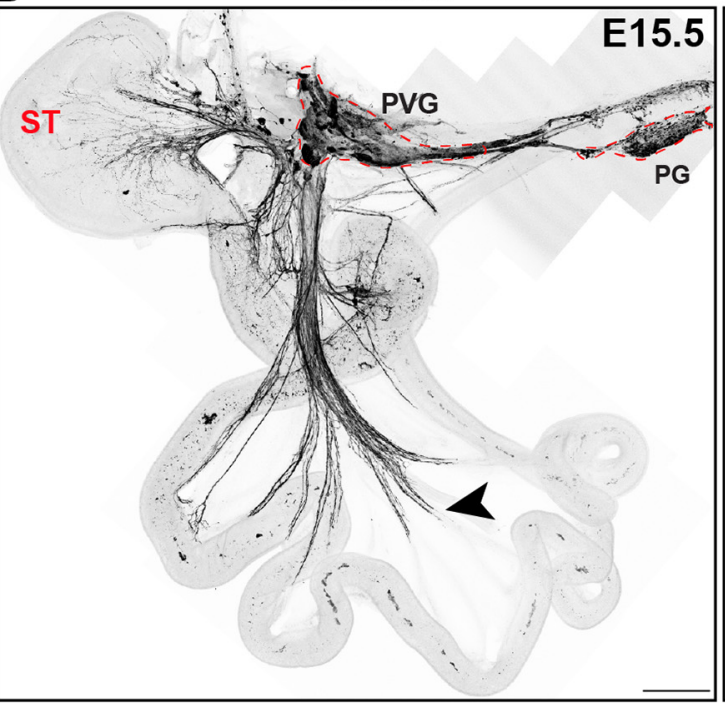

$\Delta S N$

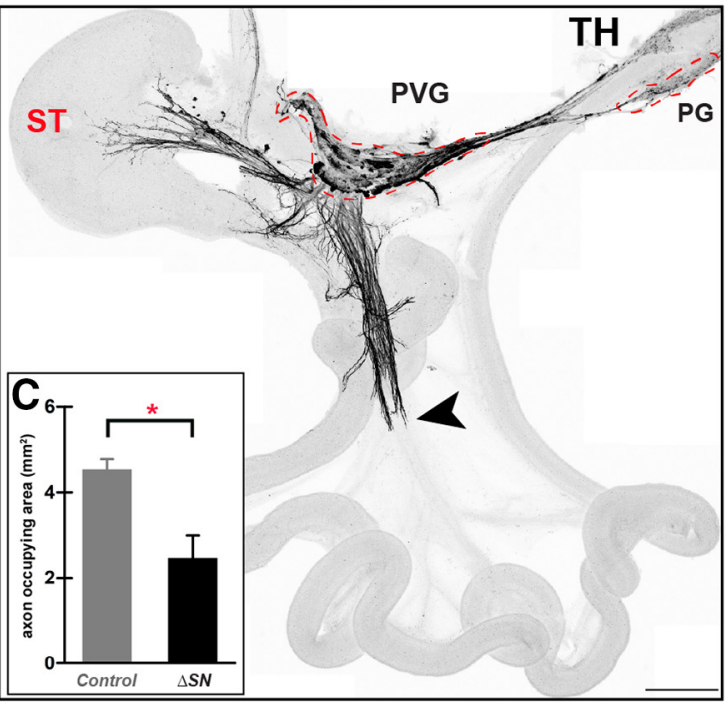

D

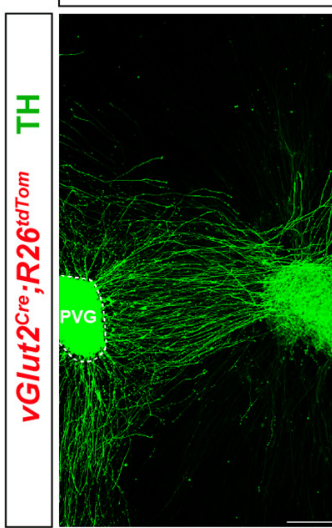

PVG + NG
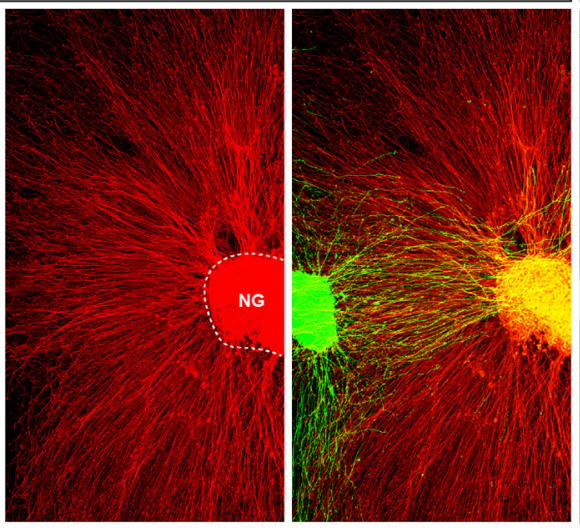

sympathetic axon (PVG)

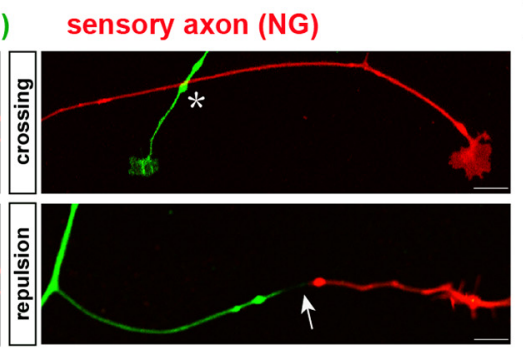

PVG + DRG

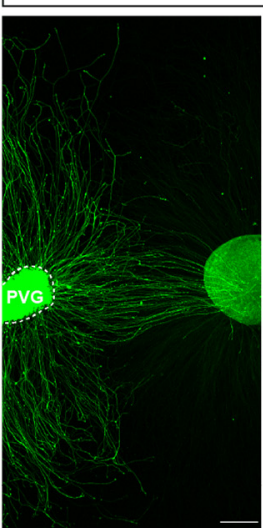

$\mathbf{F}$

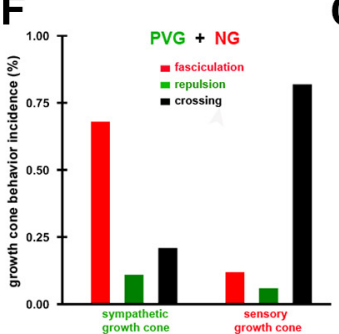

G

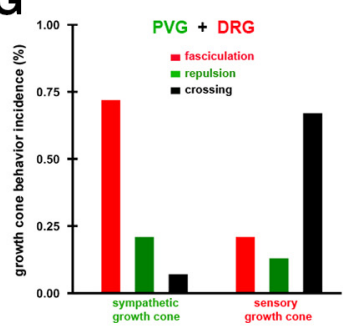

Figure 10. Attractive effect of sensory axons on sympathetic axons. $A$, Transversal sections of E14.5 Control $(n=3)$ and $\Delta S N\left(v G l u t 2^{\text {(re }} ; R 26^{D T A} ; n=3\right)$ embryos showing the great reduction of DRG sensory neurons in $\triangle S N$ embryo. $\boldsymbol{B}$, Whole-mount views of the gut from E15.5 Control $(n=3)$ and $\Delta S N(n=3)$ embryos with TH staining. Partial removal of sensory axons results in delayed extension of sympathetic axons. Arrowheads indicate pioneer sympathetic axons. C, Quantification of the total fluorescence intensity of PVG-arising TH $\mathrm{TH}^{+}$axons $(n=3$ for each group; Kruskal-Wallis test, ${ }^{*} p=0.0348$ ). D, PVG-NG and PVG-DRG explant coculture assay showing heterotypic sympathetic-sensory axonal interactions. Note: more sympathetic axons accumulating within the NG and DRG explants. $\boldsymbol{E}$, Representative examples of three types of sympathetic growth cone behaviors, including fasciculation (arrowheads), crossing (asterisk), and repulsion (arrow), on encounter with interjecting sensory axons. $\boldsymbol{F}, \boldsymbol{G}$, Quantification of the percentages of three types of growth cone behaviors. Scale bars: $100 \mu \mathrm{m}(\boldsymbol{A}), 500 \mu \mathrm{m}(\boldsymbol{B}), 200 \mu \mathrm{m}(\boldsymbol{D})$, $10 \mu \mathrm{m}(\boldsymbol{E})$. SP, spinal cord; ST, stomach; DRG, dorsal root ganglion; SG, sympathetic ganglion; PVG, prevertebral ganglion; PG, pelvic ganglion.

\section{Discussion}

Our work provides the basic information to unravel the mechanisms governing gut-innervating extrinsic axonal patterning on the following aspects: (1) characterization of intrinsic markers for various types of extrinsic axons (Table 1), thus making manipulation or tracing of extrinsic axons into the brain or the gut possible; (2) mapping of spatiotemporal extrinsic innervation of the GI tract (Fig. 6I), which is crucial to understand how the gut-brain neural circuits are assembled; and (3) revealing the roles of heterotypic axonal interactions in the gut-innervating axonal patterns (Fig. 11).

\section{Visualizing visceral sensory axons with intrinsic markers}

The limitation of intrinsic markers targeting visceral sensory neurons might attribute to the following reasons: (1) overlapping markers between sensory neurons and sympathetic or parasympathetic neurons; (2) temporal dynamics of marker genes over development; and (3) subtype diversity of visceral sensory neurons with distinctive molecular expression profiles. Brn $3 a^{\text {tau-laz, }}$ $v$ Glut $^{C r e}$, and Advillin ${ }^{\text {Cre }}$ are currently available mouse lines to generally label or manipulate sensory neurons during embryonic developmental stage (Trieu et al., 2003; Zurborg et al., 2011; Chang et al., 2015). Brn3a has been previously reported to be 

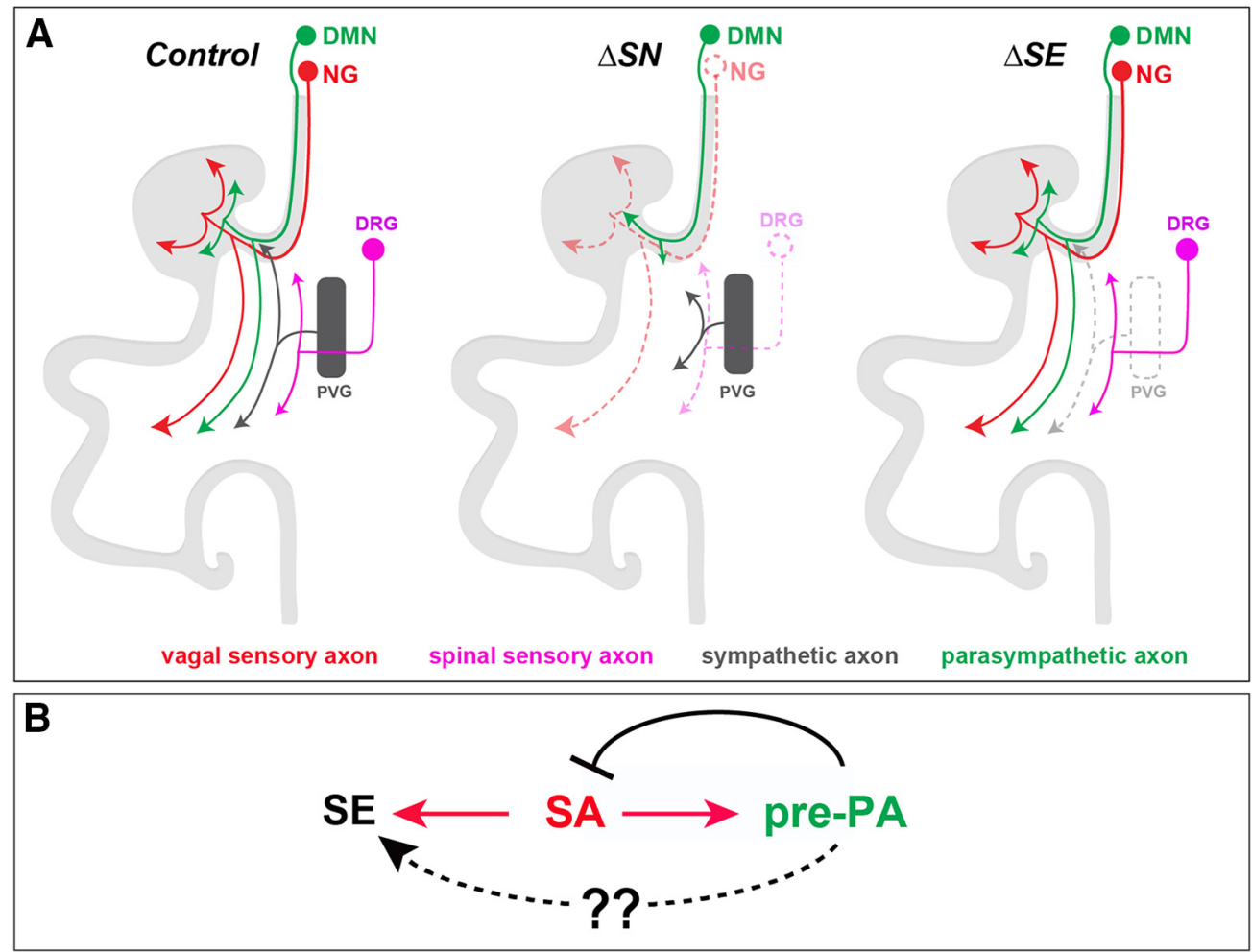

Figure 11. Hierarchical relationships among three types of extrinsic gut-innervating axons. A, Schematic: erratic pre-PA and sympathetic axonal projections in the absence of sensory trajectories $(\triangle S M)$, and unchanged sensory and pre-PA axonal projections in the absence of sympathetic trajectories ( $\triangle S E$ ). $B$, Relationships among $S A$ (sensory afferent), SE (sympathetic efferent), and pre-PA (preganglionic parasympathetic efferent) axons. SAs provide a scaffold to attractively route later-extending pre-PAs and SEs. The repulsive effect of pre-PAs on SAs is preliminarily observed in DMN-NG coculture assay in vitro, and whether pre-PAs influence SEs remains unresolved.

exclusively expressed in somatic sensory neurons, but not in visceral sensory neurons (D'Autreaux et al., 2011). By comparing the labeling efficiency of $v$ Glut $2^{\text {Cre }} ; R_{2} 6^{\text {tdTom }}$ and Advillin ${ }^{\text {Cre }}$; $R 26^{\text {tdTom }}$ embryos, our results showed that $v$ Glut $2^{\text {Cre }} ; R 26^{\text {tdTom }}$ mice are more sensitive and specific for sensory neurons during early development. Meanwhile, recent studies showed that, in Advillin ${ }^{\text {Cre }} ; R_{2} 6^{\text {tdTom }}$ adult mice, tdTom fluorescence is observed exclusively in IB4 ${ }^{+}$non-peptidergic sensory neurons (Chuang et al., 2018), as well as autonomic neurons (Hunter et al., 2018), potentially limiting its application to specifically target sensory neurons. Moreover, given that our results surprisingly identified genetically labeled vagal sensory neurons in both $\mathrm{Dbh}^{\mathrm{Cre}}$; $R 26^{\text {tdTom }}$ and Chat ${ }^{\mathrm{Cre}} ; \mathrm{R}_{2} 6^{\text {tdTom }}$ embryos, which have been widely used to trace sympathetic and parasympathetic neurons, respectively (Stanke et al., 2006; Gautron et al., 2013), caution must be applied when using these two lines to trace visceral efferent axons.

Comprising $\sim 7 \%$ of sensory neurons within the DRG, spinal visceral sensory neurons demonstrate distinctive physical features from spinal somatic and vagal visceral sensory neurons (Collman et al., 1992). In contrast to vagal sensory neurons that could be easily genetically manipulated in adult animals (Hockley et al., 2019), the scattered distribution of spinal sensory neurons within the segmental DRGs has greatly increased the technical challenges to characterize their molecular features. In this study, while we separately visualized spinal sensory axons from vagal sensory axons by co-detection with TrkA on Phox $2 b^{F L P} ; v G l u t 2^{C r e} ; R 26^{F L T G}$ embryos, the expression of TrkA in somatic sensory and enteric neurons restrains using TrkA to trace visceral spinal axonal synaptic partners within the spinal cord and gut (Hoehner et al., 1996; Lallemend and Ernfors, 2012). Gene expression profiling of adult NG and DRG has shown that some $\mathrm{G}$ protein-coupled receptors and ion channels are distinctively expressed by vagal and spinal sensory neurons (Peeters et al., 2006). Still, specific intrinsic markers to distinguish spinal and vagal sensory neurons are waiting to be identified. Single-cell RNA sequencing of retrogradely labelled gut-innervating sensory neurons offers one potential strategy to identify discriminative marker genes for visceral spinal sensory neurons (Hockley et al., 2019).

\section{Extrinsic afferent innervation of the developing GI tract}

Because of the tiny size of the NG, previous tracing experiments to map the timeline of gut-innervating vagal sensory axons were only performed in older embryos (Sang and Young, 1998; Ratcliffe et al., 2006). With anterograde and retrograde neuronal tracers, the spatiotemporal extension of vagal axons has been preliminarily documented in mice and rats (Rinaman and Levitt, 1993; Uesaka et al., 2016). In mice, it was found that vagal axons bypass the NG at E9.5, reach the esophagogastric junction at E10.0, enter the stomach at E11.0, and innervate the duodenum by E14.0 (Baetge and Gershon, 1989; Ratcliffe et al., 2006, 2011). This is highly consistent with our current findings that vagal sensory axons initiate their extension at E10.5, enter the stomach at E11.5, converge with spinal sensory axons at E12.5, and project into the gut by E16.5. Moreover, in line with the observation in rats that vagal sensory outgrowth to the gut occurs before vagal motor axons (Rinaman and Levitt, 1993), we similarly revealed that vagal sensory project first to establish an initial grid for later-extending pre-PA axons.

Despite its obvious physiological importance, relatively little information is available regarding the mechanisms controlling vagal afferent innervation of the GI tract. Considering that vagal 
sensory axons project along the migratory pathways of NCCs, it is conceivable that NCCs or NCCs-derived molecules might guide the initial sensory axonal extension from the NG to the esophagus and stomach (Baetge and Gershon, 1989; EspinosaMedina et al., 2017), but convincing evidence with detailed mechanisms is still missing. Gut-derived guidance molecules, including Netrins, neurotrophins, and Slits, have been shown to play roles in guiding vagal sensory axons to their GI destinations (Peeters et al., 2006; Ratcliffe et al., 2006; Murphy and Fox, 2010; Goldberg et al., 2013). Based on their nerve endings within the gut wall, extrinsic sensory neurons have been classified into five classes, and each class possesses distinct physiological properties (Brookes et al., 2016), implying the potential involvement of intestinal cell-derived signals in afferent axonal patterning within the gut.

Extrinsic sympathetic innervation of the developing GI tract As norepinephrine is the primary neurotransmitter for postganglionic sympathetic neurons, $D b h^{C r e}$ mice have been crossed with various reporter lines to genetically visualize sympathetic axons (Udit and Gautron, 2013). However, our results revealed that a subpopulation of sensory neurons within the NG of $D b h^{C r e} ; R 26^{t d T o m}$ embryo is genetically labeled, thus making it impossible to distinguish vagal sensory and sympathetic axons. With TH immunodetection to trace sympathetic axons, we show that sympathetic axons arise from the PVGs and PGs, project along the gut mesentery, and innervate the whole gut from E12.5 to E16.5. Interestingly, while a subpopulation of enteric neuron precursors transiently expresses catecholaminergic properties from E9.5 to E12.5 (Gershon et al., 1984; Baetge et al., 1990; Young et al., 2002; Obermayr et al., 2013b), enteric $\mathrm{TH}^{+}$neurons are rarely detected in the gut at E14.5 when sympathetic axons project into the gut, therefore allowing tracing sympathetic axons with $\mathrm{TH}$ immunodetection within the gut.

Although a variety of molecules have been shown to play roles in sympathetic axon initiation and final target innervation (Glebova and Ginty, 2005), insights into the mechanisms underlying proximal and distal sympathetic axonal extension remain sparse. Arterial vasculature has been implicated to guide sympathetic axons to their final targets (Makita et al., 2008), and vascular-derived Artemin has been identified to be the key molecule (Honma et al., 2002). However, the requirement of artemin for early neuroblast migration indicated that the deficient sympathetic projection pattern observed in artemin-/- embryos might be secondary to improper migration of neuroblast (Glebova and Ginty, 2005). Our previous study revealed that the aberrant sensory projections resulting from motor neuron elimination are precisely mirrored by sympathetic axons, therefore strongly implicating the involvement of heterotypic axonal interaction in regulating proximal extension of sympathetic axons (Wang et al., 2014). In the present study, we found that genetic ablation of sensory projections triggered a mild, but significant, delayed extension of sympathetic axons. The attractive effect of sensory axons on sympathetic axons was further confirmed with an in vitro NG-PVG and NG-DRG coculture assay. Herein, heterotypic sensory-sympathetic axonal interaction seems to play more critical roles in patterning sympathetic trajectories within the gut mesentery. Interestingly, according to its structure, function, and role in diseases, the gut mesentery has been recently reclassified as an independent organ (Coffey and O'Leary, 2016). We speculate that molecules from the gut mesentery might play additional roles to guide sympathetic axonal projections. Of note, we revealed that, instead of projecting perpendicularly along the arteries to the lumen of the gut, sympathetic axons, together with sensory and parasympathetic fibers, extended along the myenteric plexus and kept close contact with enteric neurons. Thus, signals derived from enteric neurons might also contribute to patterning sympathetic axonal projections within the gut.

\section{Extrinsic parasympathetic innervation of the developing GI} tract

In spite that previous studies have made great efforts to trace pre-PA axon with different extrinsic tracers, because of the lack of specific markers to distinguish vagal sensory axons and pre$\mathrm{PA}$ axons along the vagus nerves, the detailed information of the development of pre-PA innervation of the GI tract is still missing. Chat ${ }^{C r e} ; R 26^{\text {tdTom }}$ and Chat ${ }^{G F P}$ reporter lines have been used to detect cholinergic neurons, including parasympathetic and enteric neurons. However, the presence of both $\mathrm{tdTom}^{+}$and GFP ${ }^{+}$ neurons within the NG of Chat ${ }^{C r e} ; R_{2} 6^{\text {tdTom }}$;Chat ${ }^{\text {GFP }}$ embryos restricts its use to distinguish sensory and pre-PA axons, especially during embryonic development. While VAChT immunoreactivity is detected in both sympathetic and pre-PA neurons, absence of TH immunoreactivity in pre-PA axons makes it possible to distinguish sympathetic and pre-PA axons with VAChT and $\mathrm{TH}$ double immunodetections.

Apart from interacting with guidance cues distributed along their projection pathways, head pre-PA axons are found to project along visceral afferent axons (Coppola et al., 2010). In line with this, our data showed that the dependence of pre-PA axonal extension on visceral sensory trajectories is conserved in the vagus nerves. NG-arising sensory axons actively navigate to the GI tract by responding to axon guidance molecules distributing along their projection pathways to or within their targeting organs (Ratcliffe et al., 2006; Murphy and Fox, 2010), to establish an initial grid for later-extending parasympathetic axons. In the somatic nervous system, somatic afferent and efferent axons mutually interact with each other to ensure the coordinated projections of motor and sensory axons (Gallarda et al., 2008; Wang et al., 2011). Similarly, strong repulsive effect of DMN-arising parasympathetic axons on NG sensory axons was observed in DMN-NG coculture assay (XY.N and L.W unpublished data). Thus, mutual heterotypic axonal interactions appear to be critical to determine the long-distance projection pattern of vagus nerves. By contrast, sympathetic axons seem to have no obvious effect on parasympathetic axons as pre-PA axons demonstrated comparable normal projection patterns after the removal of most sympathetic trajectories. Thus, axonal interactions play distinctive roles in patterning various extrinsic axonal projections, but the corresponding underlying molecular mechanisms remain to be addressed.

\section{Neural connections between the gut and the brain}

Neural control of the GI tract is exerted by the neural circuit in the GBA, which is composed of three basic parts: the CNS, the ENS, and the peripheral nervous system (PNS) bridging the ENS and the CNS. In contrast to advances made in uncovering neural connections between the PNS and CNS, relatively little is known about the mechanisms underlying how neural assembly is integrated between the PNS and the ENS. The whole gut is innervated by extrinsic axons between E9.5 and E16.5, the stages that encompass the intrinsic colonization of the gut by enteric neurons. Meanwhile, as the most abundant neurons in the ENS, cholinergic neurons appear within the mouse guts at E11.0 (Rothman and Gershon, 1982; Hao et al., 2013; Erickson et al., 2014), the stage when vagal sensory and pre-PA axons enter the 
stomach. Given that neurotransmitters have been shown to play roles in regulating the differentiation of enteric neurons ( $\mathrm{Li}$ et al., 2011), it is likely that extrinsic and intrinsic axons might reciprocally regulate each other's development. In our experiments, we found that extrinsic axons project along the myenteric plexus and keep intimate contact with enteric neurons, further implicating that extrinsic axon-releasing neurotransmitters might contribute to the differentiation of enteric neurons. Gershon and Thompson reported that ACh-mediating muscle contractile responses were detected in mouse gut at E16.0 (Gershon and Thompson, 1973), when extrinsic axons innervate along the whole GI tract, raising the possibility that parasympathetic axons might be the origins of ACh within the smooth muscle. While direct evidence for these speculations is missing, our anatomic observations will provide an entry point to coordinately investigate the roles of intrinsic and extrinsic axonal interactions in the regulating GI functions.

The ENS in mature mammals contains approximately twenty types of enteric neurons, and virtually every class of neurotransmitter found in the CNS and PNS exists in the ENS (Hao and Young, 2009). Thus, genetically engineered mice, together with optogenetic technology, mono-trans-synaptic viral tools, and electrophysiology to trace or manipulate a population of neurons along the GBA, will provide potential strategies to uncover the neural circuits between the brain and the gut. Continued research efforts to understand mechanisms underlying the assembly of neural circuitry in the GBA will shed light on developing novel therapeutic strategies for GBA-related diseases including visceral pain, functional GI disorders, and mental diseases.

\section{References}

Arvidsson U, Riedl M, Elde R, Meister B (1997) Vesicular acetylcholine transporter (VAChT) protein: a novel and unique marker for cholinergic neurons in the central and peripheral nervous systems. J Comp Neurol 378:454-467.

Baetge G, Gershon MD (1989) Transient catecholaminergic (TC) cells in the vagus nerves and bowel of fetal mice: relationship to the development of enteric neurons. Dev Biol 132:189-211.

Baetge G, Pintar JE, Gershon MD (1990) Transiently catecholaminergic (TC) cells in the bowel of the fetal rat: precursors of noncatecholaminergic enteric neurons. Dev Biol 141:353-380.

Brookes S, Chen N, Humenick A, Spencer NJ, Costa M (2016) Extrinsic sensory innervation of the gut: structure and function. Adv Exp Med Biol 891:63-69.

Brookes SJ, Spencer NJ, Costa M, Zagorodnyuk VP (2013) Extrinsic primary afferent signalling in the gut. Nat Rev Gastroenterol Hepatol 10:286-296.

Browning KN, Travagli RA (2014) Central nervous system control of gastrointestinal motility and secretion and modulation of gastrointestinal functions. Compr Physiol 4:1339-1368.

Chang RB, Strochlic DE, Williams EK, Umans BD, Liberles SD (2015) Vagal sensory neuron subtypes that differentially control breathing. Cell 161: 622-633.

Chuang YC, Lee CH, Sun WH, Chen CC (2018) Involvement of advillin in somatosensory neuron subtype-specific axon regeneration and neuropathic pain. Proc Natl Acad Sci USA 115:E8557-E8566.

Cioni JM, Wong HH, Bressan D, Kodama L, Harris WA, Holt CE (2018) Axon-axon interactions regulate topographic optic tract sorting via CYFIP2-dependent WAVE complex function. Neuron 97:1078-1093.e6.

Coffey JC, O'Leary DP (2016) The mesentery: structure, function, and role in disease. Lancet Gastroenterol Hepatol 1:238-247.

Collman PI, Tremblay L, Diamant NE (1992) The distribution of spinal and vagal sensory neurons that innervate the esophagus of the cat. Gastroenterology 103:817-822.

Coppola E, Rallu M, Richard J, Dufour S, Riethmacher D, Guillemot F, Goridis C, Brunet JF (2010) Epibranchial ganglia orchestrate the development of the cranial neurogenic crest. Proc Natl Acad Sci USA 107:2066-2071.

D’Autreaux F, Coppola E, Hirsch MR, Birchmeier C, Brunet JF (2011) Homeoprotein Phox $2 \mathrm{~b}$ commands a somatic-to-visceral switch in cranial sensory pathways. Proc Natl Acad Sci USA 108:20018-20023.

Dinan TG, Cryan JF (2017) Gut-brain axis in 2016: brain-gut-microbiota axis - mood, metabolism and behaviour. Nat Rev Gastroenterol Hepatol 14:69-70.

Duong CV, Geissen M, Rohrer H (2002) The developmental expression of vasoactive intestinal peptide (VIP) in cholinergic sympathetic neurons depends on cytokines signaling through LIFRbeta-containing receptors. Development 129:1387-1396.

Erickson CS, Lee SJ, Barlow-Anacker AJ, Druckenbrod NR, Epstein ML, Gosain A (2014) Appearance of cholinergic myenteric neurons during enteric nervous system development: comparison of different ChAT fluorescent mouse reporter lines. Neurogastroenterol Motil 26:874-884.

Espinosa-Medina I, Jevans B, Boismoreau F, Chettouh Z, Enomoto H, Müller T, Birchmeier C, Burns AJ, Brunet JF (2017) Dual origin of enteric neurons in vagal Schwann cell precursors and the sympathetic neural crest. Proc Natl Acad Sci USA 114:11980-11985.

Gallarda BW, Bonanomi D, Müller D, Brown A, Alaynick WA, Andrews SE, Lemke G, Pfaff SL, Marquardt T (2008) Segregation of axial motor and sensory pathways via heterotypic trans-axonal signaling. Science 320:233-236.

Gautron L, Rutkowski JM, Burton MD, Wei W, Wan Y, Elmquist JK (2013) Neuronal and nonneuronal cholinergic structures in the mouse gastrointestinal tract and spleen. J Comp Neurol 521:3741-3767.

Gershon MD, Thompson EB (1973) The maturation of neuromuscular function in a multiply innervated structure: development of the longitudinal smooth muscle of the foetal mammalian gut and its cholinergic excitatory, adrenergic inhibitory, and non-adrenergic inhibitory innervation. J Physiol 234:257-277.

Gershon MD, Rothman TP, Joh TH, Teitelman GN (1984) Transient and differential expression of aspects of the catecholaminergic phenotype during development of the fetal bowel of rats and mice. J Neurosci 4:2269-2280.

Glebova NO, Ginty DD (2005) Growth and survival signals controlling sympathetic nervous system development. Annu Rev Neurosci 28:191-222.

Goldberg D, Borojevic R, Anderson M, Chen JJ, Gershon MD, Ratcliffe EM (2013) Slit/Robo-mediated chemorepulsion of vagal sensory axons in the fetal gut. Dev Dyn 242:9-15.

Han W, Tellez LA, Perkins MH, Perez IO, Qu T, Ferreira J, Ferreira TL, Quinn D, Liu ZW, Gao XB, Kaelberer MM, Bohórquez DV, ShammahLagnado SJ, de Lartigue G, de Araujo IE (2018) A neural circuit for gutinduced reward. Cell 175:887-888.

Hao MM, Bornstein JC, Young HM (2013) Development of myenteric cholinergic neurons in ChAT-Cre; R26R-YFP mice. J Comp Neurol 521:3358-3370.

Hao MM, Young HM (2009) Development of enteric neuron diversity. J Cell Mol Med 13:1193-1210.

Hasegawa H, Abbott S, Han BX, Qi Y, Wang F (2007) Analyzing somatosensory axon projections with the sensory neuron-specific Advillin gene. J Neurosci 27:14404-14414.

Hatch J, Mukouyama YS (2015) Spatiotemporal mapping of vascularization and innervation in the fetal murine intestine. Dev Dyn 244:56-68.

Hirsch MR, D'Autreaux F, Dymecki SM, Brunet JF, Goridis C (2013) A Phox2b::FLPo transgenic mouse line suitable for intersectional genetics. Genesis 51:506-514

Hockley JRF, Taylor TS, Callejo G, Wilbrey AL, Gutteridge A, Bach K, Winchester WJ, Bulmer DC, McMurray G, Smith ESJ (2019) Single-cell RNAseq reveals seven classes of colonic sensory neuron. Gut 68:633-644.

Hoehner JC, Wester T, Påhlman S, Olsen L (1996) Localization of neurotrophins and their high-affinity receptors during human enteric nervous system development. Gastroenterology 110:756-767.

Honma Y, Araki T, Gianino S, Bruce A, Heuckeroth R, Johnson E, Milbrandt $\mathrm{J}$ (2002) Artemin is a vascular-derived neurotropic factor for developing sympathetic neurons. Neuron 35:267-282.

Hunter DV, Smaila BD, Lopes DM, Takatoh J, Denk F, Ramer MS (2018) Advillin is expressed in all adult neural crest-derived neurons. eNeuro 5: ENEURO.0077-18.2018.

Imai T, Yamazaki T, Kobayakawa R, Kobayakawa K, Abe T, Suzuki M, Sakano H (2009) Pre-target axon sorting establishes the neural map topography. Science 325:585-590. 
Kaelberer MM, Buchanan KL, Klein ME, Barth BB, Montoya MM, Shen X, Bohórquez DV (2018) A gut-brain neural circuit for nutrient sensory transduction. Science 361:eaat5236.

Keast JR (1999) Unusual autonomic ganglia: connections, chemistry, and plasticity of pelvic ganglia. Int Rev Cytol 193:1-69.

Lallemend F, Ernfors P (2012) Molecular interactions underlying the specification of sensory neurons. Trends Neurosci 35:373-381.

Langley JN (1921) The autonomic nervous system. Part 1. Cambridge: Heffer and Sons Ltd.

Lee SK, Jurata LW, Funahashi J, Ruiz EC, Pfaff SL (2004) Analysis of embryonic motoneuron gene regulation: derepression of general activators function in concert with enhancer factors. Development 131:3295-3306.

Li Z, Chalazonitis A, Huang YY, Mann JJ, Margolis KG, Yang QM, Kim DO, Côté F, Mallet J, Gershon MD (2011) Essential roles of enteric neuronal serotonin in gastrointestinal motility and the development/survival of enteric dopaminergic neurons. J Neurosci 31:8998-9009.

Lindsay RM, Rohrer H (1985) Placodal sensory neurons in culture: nodose ganglion neurons are unresponsive to NGF, lack NGF receptors but are supported by a liver-derived neurotrophic factor. Dev Biol 112:30-48.

Makita T, Sucov HM, Gariepy CE, Yanagisawa M, Ginty DD (2008) Endothelins are vascular-derived axonal guidance cues for developing sympathetic neurons. Nature 452:759-763.

Murphy MC, Fox EA (2007) Anterograde tracing method using DiI to label vagal innervation of the embryonic and early postnatal mouse gastrointestinal tract. J Neurosci Methods 163:213-225.

Murphy MC, Fox EA (2010) Mice deficient in brain-derived neurotrophic factor have altered development of gastric vagal sensory innervation. J Comp Neurol 518:2934-2951.

Obermayr F, Hotta R, Enomoto H, Young HM (2013a) Development and developmental disorders of the enteric nervous system. Nat Rev Gastroenterol Hepatol 10:43-57.

Obermayr F, Stamp LA, Anderson CR, Young HM (2013b) Genetic fatemapping of tyrosine hydroxylase-expressing cells in the enteric nervous system. Neurogastroenterol Motil 25:e283-e291.

Parlato R, Otto C, Begus Y, Stotz S, Schütz G (2007) Specific ablation of the transcription factor CREB in sympathetic neurons surprisingly protects against developmentally regulated apoptosis. Development 134:16631670.

Peeters PJ, Aerssens J, de Hoogt R, Stanisz A, Göhlmann HW, Hillsley K, Meulemans A, Grundy D, Stead RH, Coulie B (2006) Molecular profiling of murine sensory neurons in the nodose and dorsal root ganglia labeled from the peritoneal cavity. Physiol Genomics 24:252-263.

Plummer NW, Evsyukova IY, Robertson SD, de Marchena J, Tucker CJ, Jensen P (2015) Expanding the power of recombinase-based labeling to uncover cellular diversity. Development 142:4385-4393.

Powley TL, Spaulding RA, Haglof SA (2011) Vagal afferent innervation of the proximal gastrointestinal tract mucosa: chemoreceptor and mechanoreceptor architecture. J Comp Neurol 519:644-660.

Rao M, Gershon MD (2018) Enteric nervous system development: what could possibly go wrong? Nat Rev Neurosci 19:552-565.

Ratcliffe EM, Setru SU, Chen JJ, Li ZS, D’Autreaux F, Gershon MD (2006) Netrin/DCC-mediated attraction of vagal sensory axons to the fetal mouse gut. J Comp Neurol 498:567-580.
Ratcliffe EM, Fan L, Mohammed TJ, Anderson M, Chalazonitis A, Gershon MD (2011) Enteric neurons synthesize netrins and are essential for the development of the vagal sensory innervation of the fetal gut. Dev Neurobiol 71:362-373.

Rinaman L, Levitt P (1993) Establishment of vagal sensorimotor circuits during fetal development in rats. J Neurobiol 24:641-659.

Rothman TP, Gershon MD (1982) Phenotypic expression in the developing murine enteric nervous system. J Neurosci 2:381-393.

Sang Q, Young HM (1998) The origin and development of the vagal and spinal innervation of the external muscle of the mouse esophagus. Brain Res 809:253-268.

Stanke M, Duong CV, Pape M, Geissen M, Burbach G, Deller T, Gascan H, Otto C, Parlato R, Schütz G, Rohrer H (2006) Target-dependent specification of the neurotransmitter phenotype: cholinergic differentiation of sympathetic neurons is mediated in vivo by gp 130 signaling. Development 133:141-150.

Tan LL, Bornstein JC, Anderson CR (2010) The neurochemistry and innervation patterns of extrinsic sensory and sympathetic nerves in the myenteric plexus of the C57Bl6 mouse jejunum. Neuroscience 166:564-579.

Tassicker BC, Hennig GW, Costa M, Brookes SJ (1999) Rapid anterograde and retrograde tracing from mesenteric nerve trunks to the guinea-pig small intestine in vitro. Cell Tissue Res 295:437-452.

Trieu M, Ma A, Eng SR, Fedtsova N, Turner EE (2003) Direct autoregulation and gene dosage compensation by POU-domain transcription factor Brn3a. Development 130:111-121.

Udit S, Gautron L (2013) Molecular anatomy of the gut-brain axis revealed with transgenic technologies: implications in metabolic research. Front Neurosci 7:134

Uesaka T, Young HM, Pachnis V, Enomoto H (2016) Development of the intrinsic and extrinsic innervation of the gut. Dev Biol 417:158-167.

van den Pol AN, Yao Y, Fu LY, Foo K, Huang H, Coppari R, Lowell BB, Broberger C (2009) Neuromedin B and gastrin-releasing peptide excite arcuate nucleus neuropeptide $\mathrm{Y}$ neurons in a novel transgenic mouse expressing strong Renilla green fluorescent protein in NPY neurons. J Neurosci 29:4622-4639.

Wang FB, Powley TL (2007) Vagal innervation of intestines: afferent pathways mapped with new en bloc horseradish peroxidase adaptation. Cell Tissue Res 329:221-230.

Wang L, Marquardt T (2012) Direct live monitoring of heterotypic axonaxon interactions in vitro. Nat Protoc 7:351-363.

Wang L, Marquardt T (2013) What axons tell each other: axon-axon signaling in nerve and circuit assembly. Curr Opin Neurobiol 23:974-982.

Wang L, Klein R, Zheng B, Marquardt T (2011) Anatomical coupling of sensory and motor nerve trajectory via axon tracking. Neuron 71:263-277.

Wang L, Mongera A, Bonanomi D, Cyganek L, Pfaff SL, Nüsslein-Volhard C, Marquardt T (2014) A conserved axon type hierarchy governing peripheral nerve assembly. Development 141:1875-1883.

Young HM, Jones BR, McKeown SJ (2002) The projections of early enteric neurons are influenced by the direction of neural crest cell migration. J Neurosci 22:6005-6018.

Zurborg S, Piszczek A, Martínez C, Hublitz P, Al Banchaabouchi M, Moreira P, Perlas E, Heppenstall PA (2011) Generation and characterization of an Advillin-Cre driver mouse line. Mol Pain 7:66 